\title{
Studien zum historischen Atlas der österreichischen Alpenländer.
}

\author{
Von \\ Wilhelm Erben.
}

Über den historischen Atlas der österreichischen Alpenländer hat sich im Laufe von fünfzehn Jahren schon eine besondere Literatur angesammelt. Seinen Gedanken vorzubereiten und die nötigen Kräfte zu gewinnen, hatte Eduard Richter seit 1895 mehrmals das Wort ergriffen, und sobald die Arbeit in Gang war, haben er und seine Mitarbeiter der wissenschaftlichen Welt über ihre Fortschritte Bericht erstattet; als dem umsichtigen und tatkräftigen Schöpfer des Werkes die Zügel entglitten, fühlten sich von nah und fern. Freunde und Schüler des unvergesslichen Mannes gedrängt, laut zu sagen was er ihnen und was er der Wissenschaft gewesen, und es war kaum eine unter den vielen seinem Andenken gewidmeten Schrifteu, die nicht auch gauz besonders des historischen Atlas gedacht hätte, in welchem Richters wissenschaftliche Art sich am deutlichsten verkörpert1); endlich hat dann wieder das Erscheinen der ersten Lieferung, die ein Jahr nach Richters Tod Oswald Redlich als nunmehriger Obmann der Atlaskommission mit seinen einleitenden Worten in die

1) Ein Verzeichnis der bis zu Anfang des Jahres 1906 erschienenen Nachrufe auf Richter bot Marek in den Mitt. der geographischen Gesellschaft in Wien 1906, $254 \mathrm{f}$.; s. auch Redlich in Mitt. des Instituts 27, $197 \mathrm{ff}$. und Sieger in dem von Bettelheim hrrgg. Biographischen Jahrbuch u. deutschen Nekrolog 10, $119 \mathrm{ff}$, wo einige Nachträge zur Liste Mareks zusammengestellt sind.

Mitteilungen XXX. 
Welt gehen liess, den Anlass gegeben, dass in geschichtlichen und in geographischen Fachzeitschriften berufene Beurteiler sich über Plan und Wert des Unternehmens aussprachen ${ }^{1}$ ).

Bei solcher Fülle von Äusserungen wird es mancher für überflüssig halten, den historischen Atlas auch an dieser Stelle ausführlich zu besprechen. Gerade für die österreichischen Geschichtsforscher, so könnte man meinen, sei es nicht an der Zeit, das aus ihrer Mitte hervorgegangene Werk zu rülımen, vielmehr wäre es ihre Pflicht das Begonnene in stiller rüstiger Arbeit zu fördern und zum Abschluss zu bringen; „der Worte“, so mag es scheinen, „sind genug gewechselt, lasst mich auch endlich Taten sehen". Es wird jedoch den Fortgang der Arbeit gewiss nicht hemmen, weun ein Unbeteiligter, der aussenstehend das Wachstum von Richters Unternehmen seit langem mit innerer Teilnahme verfolgt hat, im gegenwärtigen Augenblick sich zum Wort meldet. Handelt es sich ja doch beim historischen Atlas nicht blos um das Kartenwerk selbst, sondern auch um die beiden Arten von Beigaben, die Erläuterungen und die Abhandlungen, die in geschriebenem Wort das Kartenbild erklären und von dem Kartenbild erklärt werden; und sind doch gerade jetzt diese drei zusammengehörigen Reihen, die Karten, die Erläuterungen und die Abhandlungen, bis zu einem Punkte gediehen, der eine gemeinsame Beurteilung gestattet und erfordert ${ }^{2}$ ).

1) Vgl. Giannoni in dem Monatsblatt des Vereines für Landeskunde von Niederösterreich $1906 \mathrm{Nr} .7$ bis 9; Sieger in den Mitt. der geographischen Gesellschaft in Wien 1907, 241 bis 273; Curschmann in der Historischen Vierteljahrschrift 11, $539 \mathrm{ff}$., 12, 2 t.; Krabbo in der Historischen Ztschr. 101, $153 \mathrm{ff}$; Partsch in der Geographischen Ztschr. 14, $686 \mathrm{ff}$; W(idmann) in den Mitt. der Gesellsch. f. Salzburger Landeskunde 47, $380 \mathrm{ffi}$; Vučnik in der Ztschr. des historischen Vereines für Steiermark 4, $225 \mathrm{ff}$. $\mathrm{Zu}$ der älteren einschlägigen Literatur, welche noch Richter in der Einleitung der Erläuterungen z. hist. Atlas S. II, dann Giannoni und Sieger in ihren hier angeführten Besprechungen zusammengestellt haben, vgl. noch Beschorner in der Hist. Vierteljahrschr. 9, 18, v. Karg-Bebenburg in den Forschungen zur Gesch. Bayerns 13, $240 \mathrm{ff}$. u. Mell in den Mitt. des Inst. 28, $180 \mathrm{ff}$., die den bist. Atlas der österr. Alpenländer mit verwandten Unternehmungen $u$. Plänen vergleichen.

2) $\mathrm{H}$ istorischer Atlas der österreichischen Alpenländer herausgegeben von der kais. Akademie der Wissenschaften in Wien. I. Abteilung: Die Landgerichtskarte, bearbeitet unter der Leitung ron weiland Eduard Richter. 1. Lieferung: Salzburg (von Eduard Richter), Oberösterreich (von Julius Strnadt), Steiermark (von Anton Mell und Hans Pirchegger) [Übersichtsblatt der Landgerichtskarte und 11 Blätter 1 : 200.000, Querfolio]. Erläuterungen [IV und 49 S. Foliol. Wien 1906, Verlag von Adolf Holzhausen. - Abhandlungen z um historischen Atlas der österr. Alpenländer: I. Die Entstehung der Landgerichte im bayrisch österreichischen Rechtsgebiete von Hans von Voltelini 
I.

Überblicken wir nun zunächst den Stand des Unternehmens, so zeigt sich, dass seine drei Zweige, was ja ihrer Natur nach nicht anders zu erwarten war, auch jetzt keineswegs nach allen Richtungen gleich stark entwickelt sind. Das erklärt sich schon aus rein äusserlichen Gründen. Die Bearbeitung der Karten musste nach den geschichtlich zusammengehörigen und darum auch archivalisch mit einander verknüpften Gebieten, also kronlandsweise erfolgen; und Hand in Hand mit ihr musste die Niederschrift der Erläuterungen gehen. Aber die Herausgabe der einzelnen Blätter, die nur nach dem Gradnetz und nicht nach dem unregelmässigen Verlauf der Grenzen von einander getrennt werden konnten, war dort, wo zwei verschiedene Kronlïnder auf einem Kartenblatt zusammentreffen, an den Abschluss beider Arbeitsgebiete gebunden, während bei den Erläuterungen kein Grund und auch keine Möglichkeit vorlag, den zu einem Kronland gehörigen Text auf zwei Lieferungen zu verteilen. So kam es, dass die erste Lieferung des Atlas zwar nach Inhalt und Titel der Erlänterungen die drei Gebiete: Salzburg, Oberösterreich und Steiermark umfasst, nach dem Umfang der Kartenblätter aber keines der drei länder gänzlich erledigt hat. Am besten ist bei dieser unvermeidlichen Verzögerung in der Hinausgabe einzelner Blätter diesmal das Land ob der Enus weggekommen, dessen Kartenbild uns nahezu abgeschlossen vorliegt, so dass nur ganz kleine Landstreifen im äussersten Osten und Westen noch ausstehen; von Steiermark fehlen der Nordosten, der mit den anstossenden Teilen von Niederösterreich zusammenhängt, und der Süden, bei dem man auf Fertigstellung der kärntnerischen und krainerischen Grenzgebiete wird warten müssen; verhältnismäßig am meisten leidet das kleine Salzburg unter den Wirkungen des Gradnetzes, da sein ganzer Westen, der Pinzgau und seine jetzt zu Baiern gehörigen Landgerichte, obwohl längst von

(Archir für österr. Geschichte 94. Bd. S. 1 bis 40), II. Immunität, Landeshobeit und Waldschenkungen ron Eduard Kichter (ebenda S, 41 bis 62), III. Gemarkungen und Steuergemeinden im Lande Salzburg von Eduard Richter (ebenda S. 63 bis 82), IV. Das Land im Norden der Donau von Julius Strnadt, mit einer historischen Karte (ebenda S. 83 bis 310 ), V. Immunität, grund- und leibherrliche Gerichtsbarkeit in Südtirol von Hans von Voltelini (ebenda S. 311 bis 463), VI. Das Gebiet zwischen der Traun und der Ens von Julius Strnadt, mit einer Karte und einer Kartenskizze im Texte (ebenda S. 465 bis 661), VII. Hausruck und Atergan von Julius Strna dt, mit einer Karte und einer Karten. skizze im Texte (Archiv für österr. Geschichte 99. Bd. S. 1 bis 396). Wien 190 5 bis 1908, in Kommission bei Carl Gerolds Sohn und bei Alfred Hölder. 
Richter bearbeitet, auf Blätter fallen, an denen auch Nordtirol beteiligt ist. Im Ganzen sind von dem Gebiet, welches bis heute die Erläuterungen umfassen, nur etwa drei Viertel auf den gleichzeitig herausgegebenen Karten dargestellt; für den Benützer und, um es deutlicher zu sagen, für den Käufer, erwachsen daraus, wenn er vom landesgeschichtlichen Standpunkt ausgeht, unangenehme Nachteile ${ }^{1}$ ), für die Beurteilung der Frage aber, inwieweit Karten und Text ineinander greifen, sich ergänzen und erklären, genügen diese drei Viertel vollauf.

Stärkere Unterschiede ergeben sich, wenn man den Stand der Kartenpublikation oder auch den der Erläuterungen mit der bisherigen Entwicklung des dritten Zweiges des Gesamtunternehmens, mit dem der Abhandlungen vergleicht. Diese sollen nach Richters Plan und wach dem Wortlaut des Vorwortes ${ }^{2}$ ) „ausführlichere Nachweise oder Erörterungen" umfassen, die „durch die Forschungen für den Atlas. veranlasst, doch auch über die nächsten Fragen hinausreichende Probleme behandeln" und für welche die "den Karten beizugebenden Erläuterungen bei der notwendigen Knappheit ihrer Fassung nicht immer" den ausreichenden und den richtigen Platz gewähren. Unter den so gekennzeichneten Beyrift der "Abhandlungen" fallen also nicht nur Arbeiten, die der kartographischen Fixierung vorangehen, die dem Atlasarbeiter die Begriffe klären und die Wege aufhellen, sondern auch andere, welche Nebenfrüchte dieser historisch-geographischen Studien darlegen und den Atlas mit sonstigen wissenschaftlichen Richtungen in Verbindung setzen. Schon dieses weitgedehnte Programm bringt es mit sich, dass die Abhandlungen mit den Kartenblättern und ihren Erlälıterungen nicht immer gleichen Schritt halten können; manche von ihnen müssen der Bestimmung und Eintragung der Grenzen vorhergehen, andere können erst folgen, sobald die Karte fertig verliegt. Voltelinis Aufsätze gehören zu denen, welche dem weiteren Gang der kartographisehen Arbeit voraneilen und vorarbeiten; ihr Mittelpunkt ist Südtirol und zwar auch dort, wo der Verf. die Grenzen dieses. Gebietes überschreitend Nordtirol und die übrigen Teile des bairisch-

1) Vielleicht ist die Hoffnung nicht unberechtigt, dass der Verlag in Zukunft solchen Abnehmern, die sich ein Kronlandsbild vervollständigen wollen, insbesondere den Schulen und landesgeschichtlichen Vereinen, auch den Ankauf einzelner in ihr Gebiet einschlägiger Kartenblätter gestatten wird.

2) Archiv f. österr. Geschichte $94 \mathrm{~S} . \mathrm{V}$; vgl. auch Redlich in den Erläut. S. IV, eine frühere Äusserung Richters in den Deutschen Geschichtsblättern 4, 150, u. was Karg.Bebenburg in den Forscb. z. Gesch. Bayerns 13, 244 wohl auf Grund von Richters Anweisung an die Mitarbeiter anfübrt. 
österreichischen Bereichs in seine Betrachtung einbezieht; die kartographische Darstellung wird in diesem Fall erst nachträglich, in einer weiteren Lieferung des Atlas zu erfolgen haben. Hingegen stehen die übrigen fünf bisher erschienenen Abhandlungen in enger Beziehung zu den schon ausgegebeuen Kartenblättern; die beiden von Richter verfassten ergänzen und begründen die Arbeit, die im Atlas und seinen Erläuterungen für Salzburg geleistet ist; die drei übrigen führen weiter, was Strnadt an diesen beiden Stellen für Oberösterreich bietet. Freilich ist von Strnadts Abhandlungen die über das Innviertel noch ausständig; um weitere Abhandlungen Richters für Salzburg, auf die er schon an mehreren Stellen seiner Erläuterungen (S. 2, 4, 6) Bezug nimmt, hat uns sein früher Tod gebracht; Steiermark ist im Rahmen der "Abhandlungen" bisher gar nicht vertreten und man muss an anderer Stelle gedruckte Ausführungen ') heranziehen, um dafür einen Ersatz zu finden. Ist also für keines der drei in Betracht kommenden Länder derzeit die Reihe der Abhandlungen abgeschlossen, so genügen doch die bis heute vorliegenden, um sich ein Bild von dem zu machen, was die Zukunft bringen dürfte, wenn in den bisherigen Bahnen fortgefahren wird.

So ist es an der Zeit bei einer Besprechung des historischen Atlas vor allem die Frage ins Auge zu fassen, inwiefern die drei Formen, in denen uns die gewonnenen Ergebnisse dargeboten werden, Karten, Erläuterungen und Abhandlungen, richtig ineinander greifen und sich ergänzen. Diese Frage aber führt zurück zu der zweiten, ob überhaupt die Herstellung eines doppelten Textes, der Erläuterungen und der $A b h a n d l u n g e n$, richtig war und bei der Fortsetzung der Arbeit beizubehalten oder auch bei verwandten Unternehmungen nachzuahmen wäre. Die Antwort kann, so glaube ich, nur in dankbarer Anerkennung dieser Einrichtung bestehen. Der Vergleich nit den spärlichen Randbemerkungen, welche A. Schröder seiner Karte der "Herrschaftsgebiete im heutigen Regierungsbezirk

1) Mell, Der comitatus Liupoldi und dessen Aufteilung in die Landgerichte des 19. Jahrhunderts; dieser schon im J. 1900 in den Mitt. des Inst. 21, 385 ff. veröffentlichte Aufsatz diente als, Text- und Kartenprobe für den damals in Werden begriffenen Atlas und er hat in solchem Sinn wesentlich zur Klärung der methodischen Fragen beigetragen. Andere auf Steiermark, aber auch auf Kärnten u. Salzburg bezügliche Abhandlungen wurden in dem von Mell im September 1901 zu Salzburg erstatteten und in den Deutschen Geschichtsblättern 6, 54 ff. gedruckten Berichte (s. besonders S. 63 f.) in Aussicht gestellt. - Übrigens hat auch Strnadt, ehe die, Abhandlungen zum Atlas zu erscheinen anfingen, einschlägige Beiträge in den Mitt. des Inst. 23, $647 \mathrm{ff}$., 24, $433 \mathrm{ff}$. u. $646 \mathrm{ff}$. veroffentlicht; einen Nachtrag zur ersten, Abhandlung \& brachte er ebenda 27, 326 f. 
Schwaben und Neuburg“ ${ }^{*}$ beigegeben hat ${ }^{1}$ ), fällt jedenfalls durchaus zu Gunsten der österreichischen Erläuterungen aus, die weit mehr bieten, ohne unübersichtlich zu werden. Die in ihrer Art höchst wertvollen Textbände, die das rheinische Kartenwerk begleiten, haben mit den Erläuterungen nach Richters System nur den Namen gemein; sachlich stehen sie ziemlich auf einer Höhe mit den Abhandlungen, sowohl wegen ihres Umfangs ${ }^{2}$ ) wie auch insoferne, als sie von den Karten losgelöst erscheinen und fortschreiten. In Gegensatz zu ihnen geben die österreichischen Erläuterungen in knapper Zusammenfassung. diejenige Ergänzung und Aufklärung zu den zugleich erscheinenden Karten, deren der Benützer sofort bedarf. Die enge Zusammengehörigkeit von Atlas und Erläuterungen kommt in dem übereinstimmenden Format glücklich zum Ausdruck. Es entstehen dabei freilich grosse Textseiten, von denen jede bei dem engen Druck eine sehr grosse Menge von Einzeltatsachen und Belegen enthält, die Übersichtlichkeit geht dabei indes nicht verloren. Der in fetter Schrift hervortretende Namen des Landgerichtes, der nebst der Nummer des betreffenden Atlasblattes über jedem Absatz steht, ist für die Orientierung sehr förderlich. Hätte man trotzdem das Aufsuchen bestimmter Stellen und das Zitieren noch weiter erleichtern wollen, dann würde es sich vielleicht empfohlen haben, unten anstatt der Seitenzählung eine Spaltenzählung einzuführen und auch den Kopf der Seiten so zu gestalten, dass er der Übersichtlichkeit diene. Was jetzt dort steht: „Historischer Altas der österr. Alpenländer, Erläuterungen zur Landgerichtskarte", das ist für den Benützer so ziemlich wertlos und zunächst wohl nur als Orientierung für den Buchbinder berechnet; fände man dagegen auf jeder beim Aufschlagẹn linksliegenden Seite

1) Diese in der 7. Auft. von Dahlmann.Waitz Quellenkunde und in derer Ergbd. noch nicht verzeichnete, aber in den Rahmen der deutschen hist. Atlanten neuerer Zeit hineingehörende Karte $(1: 200.000)$ ist 1906 vom historischen Verein für Schwaben und Neuburg herausgegeben worden und zwar als Ersatz für das. 32. Heft seiner, Zeitschrift “.

2) Schulteis widmet den Karten von 1813 und 1818 im 1. Bde. der , Erläuterungen z. gesch. Atlas der Rheinprovinz" (Publikationen der Gesellsch. f. rheinische Geschichtskunde XII) 204 Seiten; Fabricius handelt in 2. Bde. über die Karte ron 1789 auf 789 Seiten, in 3. über das Hochgericht Khaunen (etwa $100 \square \mathrm{km}$ ) auf 99 Seiten (mit 6 Karten), Forst widmet im 4. Bande dem Fürstentum Prüm (etwa $395 \square \mathrm{km}$ ) 144 Seiten und 3 Karten, während Strnadt über das damit vergleichbare, aber wesentlich grössere Fürstentum Passau das Nötigste in nicht ganz zwei Spalten der Erläuterungen zusammenfasst, und auch Richters Erlëuterungen für Salzburg (im alten Umfang etwa $8000 \square \mathrm{km}$ ) auf sechs Seiten beschränkt sind. 
ded Kronlandsnamen, auf jeder rechtsseitigen eine zusammenfassende Bezeichnung für die hier behandelten Gerichte (etwa „Landgerichte im vormaligen Mühlkreis" oder "Landgerichte im Ennstal“ u. dgl.), so wäre das sebr willkommen. Vielleicht würde sich auch in der Gruppierung des Stoffes da und dort grössere Übersichtlichkeit erreichen lassen, als es geschehen ist ${ }^{1}$ ). Allein das sind Mängel der Ausführung, nicht Fehler des Systems, dessen Beibehaltung und Weiterführung man durchaus wünschen muss. Naturgemäß wird es jeden der Bearbeiter drängen, die Fülle der Arbeit und der Einzelbeobachtungen vor dem Benützer auszubreiten, die nötig war, um die Geschichte der Gerichtsbezirke klarzulegen. Aber es ist nicht das Interesse des Benützers, dass ihm die Erläuterungen dieses ganze Tatsachenmateriale vorführen. Wer in der einen Hand die Karte bält, um sich die räumliche Verteilung der Gerichte zu veranschaulichen, der kann sich, soll er das geographische Bild nicht aus dem Auge verlieren, unmöglich in alle Einzelheiten der Begründung, in Streitfragen über Deutung und Beurteilung von Urkunden, in genealogische oder

1) Strnadts Erläuterungen lassen in dieser Hinsicht einiges zu wünschen übrig. Die Überschriften sind nicht immer so gewählt, dass das Verbältnis der grossen und ursprünglichen Gerichte und der jüngeren kleineren Bildungen richtig hervorträte. Auch steht mancher Satz nicht an der richtigen Stelle. Die Ausführungen über das Ldg. Machland S. 9 f. dürften wohl durch eine nachträgliche Änderung der Disposition oder durch Verwechslung der Manuskriptb]ätter gelitten haben; die drei Absätze der \&. 9, die mit "Ldg. Waldhausen", ,Ldg. Baumgartenberg “, „Ldg. Arbing « überschrieben sind, unterbrechen den Zurammenhang zwischen den allgemeinen Erörterungen über das alte Ldg. Machland und, die erste Bresche die hinein gelegt wurde; zudem bilden sie teilweise eine Art Doublette zu dem, was S. 10, 2. Sp. im 2. Absatz gesagt ist, ohne dass von der einen Stelle auf die andere verwiesen wäre. Auch gehört, was S. 9, 2. Sp. über die Zelkingischen Untertanen und den Burgfried St. Thomas gesagt ist, doch nicht unter die Überschrift, Ldg. Baumgartenberg ", sondern zu S. 10 Ldg. Greinburg, wo dasselbe mit anderen Worten, und wieder ohne Verweis auf die frühere Stelle, nochmals gesagt ist. - Die vier Zeilen der 1. Sp. von S. 11 , Als Landschranne " bis ,im Machland " gehören natürlich nicht an diese Stelle sondern vor die Überschrift ,Landg. Mauthausen u. s. w.`. - Ein Verstoss gegen die Übersichtlichkeit ist es auch, wenn die fettgedruckten Überschriften nicht mit den Stammbäumen in Einklang gebracht sind, wenn also z. B. S. 11 eine der Absatzaberschriften den Namen Lobenstein enthält, obwohl ein so benanntes Gericht in dem Stammbaum S. 12 nicht rorkommt und ïberhaupt nicht zur Riedmark sondern zum Ldg. Wachsenberg gehört, vgl. Erläut. S. 13, 2. Sp. unten. Solche Versehen wirken doppelt störend in einem Text, der durch die starke Hervorhebung von Überschriften den Eindruck einer streng durchgeführten Ordnung erweckt. An einzelnen Stellen also wäre der Wunsch Curschmanns (Hist. Vierteljahrscbr. 11, 539) nach übersichtlicherer Disponierung des Textes wohl berechtigt. 
juristische Auseinandersetzungen einlassen: er bedarf vielmehr eines knappgefaßten Texts, der nur das erste Vorkommen oder die Entstehungszeit, die wechselnden Namen und Inhaber eines Gerichts, seine Spaltung oder Vereinigung mit anderen Gebieten kurz und mit aller zulässigen Sicherheit zusammenfasst und durch entsprechende Hinweise das Weiterverfolgen des Gegenstands ermöglicht ${ }^{1}$ ). Dazu gehören dann noch die allgemeinen Bemerkungen über die Gerichtsorganisation des ganzen Landes, welche der Aufzählung einzelner Gerichte vorangehen nüssen. Alles dies, aber auch nicht nehr als dies sollen die Erläuterungen bieten und dieses Mass ist in der vorliegenden Lieferung zumeist glücklich eingehalten. Sollte es in späteren Lieferungen überschritten werden, so wird dem Leser ein notwendiger Behelf zum Verständnis der Karten geraubt werden. Man würde die Benützung überaus erschweren, wenn man die Erläuterungen für die weiteren Kronländer breiter ausgestalten würde, als es bisher der Fall war. Ein ernster Grund, von dem gegebenen Muster abzuweichen liegt ja bei dem österreichischen Unternehmen nicht vor, da für breitere Begründung die Abhandlungen genügenden Raum bieten.

\section{II.}

Bei aller Kürze können indes auch die „Erläuterunge n" neben dei Einzelnachweisen, die der Atlas erfordert, allgemein interessante

1) Ernster Erwägung scheint mir wert zu sein, ob nicht auch der Gerichtsverpfändungen in den Erläut. regelmässig und mit Angabe von Namen und Zeit gelacht werden sollte. Ich vermisse derartige Angaben S. 5 beim Pfieggericht Mattree, wo auf meine einschlägigen Untersuchungen zwar hingewiesen, aher nicht einmal der Ort ihres Erscheinens (Fontes rer. Austr. II, 49; die im N. Archiv 22 enthaltene Veröffentlichung kommt für den Atlas nicht in Betracht), angeführt ist, noch weniger ihre Ergebnisse verwertet sind; dann bein Gericht Haunsbarg, das 1364 dem Ulrich Nussdorfer verpfändet wurde (Hauthaler in den Xenia Austriaca f. d. 42. Versammlung Deutscher Philologeu u. Schulmänner $1893=$ 44. Programm des Gymnasiums Borromäum zu Salzburg S. 25 Nr. 7); Erläut. S. 22 sind, wiederholte Verpfündungen c des Ldg. Kammer wohl erwähnt, aber nicht im einzelnen genannt. Ganz unerwähnt lässt Strnadt in den Erläut. S. 24, dass das Ldg. Wildeneck, eine der Erwerbungen Maximilians im bairischen Erbfolgekrieg, unmittelbar nachdem es von Baiern an Österreich abgetreten war, dem Salzburger Erzbischof verpfändet wurde, und dass es während der nächsten 60 Jahre von salzburgischer Seite schon wie ein bleibender Erwerb angesehen ward (Richter in den Mitt. des Instituts 1. Ergbd. 716 Anm. 1); vielleicht gibt der letzte Teil der Abhandlungen, der dem Innviertel gewidmet sein wird, Strnadt die Gelegenheit diese Dinge genauer darzustellen; sie verdienen es umsomehr, als sich die Vermutung aufdrängt, dass die bisher ungelösten Fragen nach dem Erwerb von Höchfeld-Strasswalchen und nach der Vorschiebung der salzburgischen Grenze an den Attersee, hier ihre Beantwortung finden werden. 
Ergebnisse in sich schliessen und schon die vorliegende Lieferung ist reich an solchen. Vor allem ist es höchst willkommen den Entwicklungsgang der Gerichtseinteilung überall bis ins einzelne ergründet und in den jedem Kronland gewidmeten einleitenden Bemerkungen besonders zusammengefasst zu sehen. War die weitgehende Zersplitterung der Gerichtsbezirke in den österreichischen Ländern schon früher bekannt1), so tritt sie jetzt, im einzelnen belegt und beleuchtet, doch noch viel deutlicher hervor. Der vergebliche Versuch Josefs II., die wirre Masse von Gerichtsinstanzen in einheitliche Form zu zwängen, und die Rückkehr zu den alten, nun bis in die Mitte des 19. Jahrhunderts fortgeschleppten Zuständen erhält ein lehrreiches Seitenstück in den Schicksalen der oberösterreichischen Gerichtsverfassung zur Zeit der bairischen Herrschaft in Innkreis und im Westen des Hausruckkreises ${ }^{2}$ ). Dort war durch Verfügungen von 1810 und 1812 die Patrimonialgerichtsbarkeit von Kriminal- und Streitsachen ausgeschlossen und sie sollte auch in diesem beschränkten Umfang nur soweit bleibend zugelassen werden, als die Grundobrigkeit über eineu geschlossenen Besitz verfügte. Nach der Rückerwerbung durch Österreich liess Kaiser Franz die alten Zustände wiederaufleben und gestattete, sogar über den Autrag der obersten Justizst-lle noch weiter zurückgehend, dic exemte Kriminaljurisdiktion über Untertanen in fremden Landgerichten. Diese mit Zähigkeit festgehaltenen österreichischen Verhältnisse heben sich deutlich ab von der Entwicklung in Baiern, dessen Gerichtseinteilung schon um des bis 1779 dorthin gehörigen Innviertels willen von Strnadt einbezogen werden musste, und ebenso sehr auch von denen in Salzburg. In beiden Ländern ist die Zersplitterung der Gerichtsbarkeit vermieden worden, indem die Landesherren, einmal in den Besitz der Gerichte gelangt, diese nur mehr auf Lebenszeit verliehen oder durch Beamte verwalten liessen und indem sie durch tunlichste Einschränkung der niederen Gerichtsbarkeit den Keim zur Bildung neuer Instanzen und Bezirke erstickten $^{3}$ ). Sehr za beachten ist, dass in den ersten drei Jahrzehnten des 17. Jahrhunderts, also zu einer Zeit, da im benachbarten Lande

1) Vgl. Luschin, Geschichte des älteren Gerichtswesens in Österreich ob und unter der Enns S. $112 \mathrm{ft}$.

2) Strnadt Erläut. S. 7. Ergänzungen dazu bietet derselbe im Arch. f. öst. Gesch. 94, $298 \mathrm{ff}$.

9) Richter in den Erläut. S. 1, Strnadt ebenda S. 7. Der Gegensatz tritt am schärfsten hervor, wenn man die Blätter 4 und 5 des Atlas nebeneinanderhält. Vgl. indes auch Riezler, Geschichte Bayerns 3, $701 \mathrm{ff}$., wornach auch Bayern von der Zersplitterung der Gerichtsbezirke nicht ganz verschont blieb. 
ob der Enns die Zersplitternng schon einen sehr hohen Grad erreicht hatte und immer noch weiter fortschritt1), salzburgische Erzbischöfe durch Zusammenlegung kleiner Gerichte grössere Bezirke gebildet haben: in den Jahren 1603 und 1608 vereinigte Wolf Dietrich die alten Gerichte Anthering, Haunsberg, Unterlebenau und zum mindesten einen Teil des Gerichtes Oberlebenau mit dem Stadtgericht Laufen zu einem einheitlichen Pfleggericht; unter Paris Lodron wurde in Jahre 1629 das Gericht an der Glan aufgelöst und teils zum Salzburger Stadtgericht, teils zum Gericht Stauffeneck geschlagen, welch letzteres wohl um dieselbe Zeit mit den früher selbständigen Gerichten Ober- und Unterplain zusammenschmolz ${ }^{2}$ ). Das sind Anzeichen einer bewussten Verwaltungspolitik, die zu den Vorgängen auf österreichischer Seite in scharfem Gegensatze steht. Die Erläuterungen lassen freilich die Frage nach den Motiven offen, die zu diesen Massregeln geführt haben ${ }^{3}$ ), aber sie laden zu ihrer Lösung ein und werden

1) Für Steiermark bezeichnet Pirchegger (Erläut. S. 29) namentlich die Regierung K. Friedrichs III. und dann die zweite Hälfte des 17. Jahrh. als die Zeit der Zerschlagung der grossen Landgerichte und als eine Periode weiterer Gerichtsverleihungen. Strnadt scheint für Oberösterreich keine zusammenfassenden Beobachtungen dieser Art angestellt zu haben; die Durchsicht seiner Erläuterungen und Gerichtsstammbäume macht incles den Eindruck, als ob hier die letzten Jahrzehnte des 16. Jahrhunderts die Zeit wären, in welcher die Zersplitterung die reissendsten Fortschritte machte. Der Verkauf der Herrschaften Pernstein, Kammer, Frankenburg und Kogel clurch Kaiser Rudolf If. (Erläut. S. 16 und 22) dürfte wesentlich zu der Bewegung beigetragen haben, die dann der bekannte Landeshauptmann Hans Jakob Löbl im Machland besonders eifrig fortführte (ebenda S. 10).

2) Richter in den Erläut. S. 4 bis 6, wo indess der Zeitpunkt der Vereinigung ron Stauffeneck mit den beiden Plainer Gerichten nicht genau bezeichnet ist; vgl, auch (Kleimayrn) Nachrichten von Juvavia (1784) S. 429.

3) Sowie Vereinigungen von Landgerichten in Salzburg auch sonst schon früher vorgekommen sind (s. Richter in den Erläut. S. 2, 2. Sp. unten und Sieger in den Mitt. der geographischen Gesellschaft 1907, 252 Anm. 1), so lassen sich auch urkundlich Vereinigungen einzelner von den obengenannten Gerichten schon früher nachweisen; so war Anthering wenigstens 1476 bis 1479 mit dem Stadtgericht Laufen in einer Hand (der des Johann Mülhaimer) vereinigt (Mitt. der Gesellsch. f. Salzb. Landeskunde 15b, 119, $126 \mathrm{t}$., 137 f.), in 16. Jahrh. aber mit Haunsberg (Erläut. S. 5, 1. Sp. unten). Vielleicht waren auch Ober- und Unterlebenau zeitweilig vereint, da 1464, 1490 und 1496 von Pflegern und Landrichtern in der Lebenau (zu Lebenau) ohne Unterscheidung der beiden Bezirke die Rede ist (Mitt. der Gesellsch. 15 ${ }^{\mathrm{b}}, 32 ; 16^{\mathrm{b}}, 261,318$ ) und da auch Örtlich. keiten um St. Georgen stets als im Lebenauer Gericht und nicht als im Unterlebe. nauer Gericht gelegen bezeichnet werden (viele Belege ebda. 14b, $15^{\mathrm{b}}$ u. $16^{\mathrm{b}}$ ). Die Frage der Zusammenziehung mehrerer Gerichte wird sich übrigens nicht trennen lassen von der anderen Frage, wie der Pflichtenkreis der Pfleger von dem der 
auch sonst eine Fundgrube werden für den, der die Veränderungen der Gerichtsorganisation vom Ausgang des Mittelalters bis ins 19. Jahrhundert auf ihre tieferliegenden allgemeinen Ursachen zurückzuführen sucht.

Nicht so zahlreich wie auf diesem späteren Gebiet sind die Aufschlüsse, die man von den Erläuterungen inbezug auf das frühere Mittelalter zu erwarten hat. Neue Quellen sind hier nicht mehr zu eröffnen und die Anfangs von Richter und seinen Arbeitsgenossen gehegte Hoffnung, es würden sich aus der späteren Gerichtsorganisation sichere Schlüsse auf die Einteilung der Grafschaften und Gaue ableiten lassen, ist im Lauf der Arbeit doch wesentlich abgeschwächt worden. Noch im Herbst 1904 wollte wenigstens Mell an der Durchführung des ursprünglichen Gedankens festhalten und ,auf dem Wege retrogressiver Forschung" bis zu den "ältesten Bezirken" zurückgreifen ${ }^{1}$ ). Nicht viel mehr als ein Jahr darnach gelangten aber die Leiter des Unternehmens zu dem Entschluss, von der Eintragung der Grafschaftsgrenzen in die Karten abzusehen ${ }^{2}$ ) und demgemäss tritt auch in den Erläuterungen jene Frühzeit der Gerichtsorganisation sehr in den Hintergrund gegenüber der Darlegung der späteren Verhältnisse. Pirchegger hat sich begnügt die Gerichtsverhältnisse bei Judenburg im Murtal bis zu dem landesfürstlichen Urbar von 1265 zurückzuführen uud er hat mit Recht auf alle die schwierigen und unsicheren Konstruktionen verzichtet, welche Mell angestellt hatte, um von da bis zu dem "comitatus Liupoldi" hinaufzugelangen ${ }^{3}$ ); sowie hier sind auch sonst in den Erläuterungen für .Steiermark die ungewissen älteren Glieder ausgelassen; die Gerichtsstammbäume beginnen also fast durchwegs mit dem 13. Jahrhundert. Strnadt hat allerdings in seinem vielleicht schon früher niedergeschriebenen Anteil der Erläuterungen an dem ursprünglichen Plane noch festgehalten, die Mehrzahl seiner Stammbäume erweckt den Anschein, als ob sich die späteren Gerichtseinteilungen in die Grafenzeit zurückführen liessen, und an mehr als

ihnen teilweise untergeordneten Landrichter geschieden war. Einige Bemerkungen darüber bot Seraphine Puchleitner im 3. Jahresbericht des städtischen Mädchenlyzeums in Brünn 1903|4 S. 8; die von ihr benützten Bestallungsbücher des 16. und 17. Jahrhts. lassen wohl noch genauere Aufschlüsse in dieser Kichtung erhoffen.

1) Deutsche Geschichtsblätter 6, 62.

2) Redlich in den Erläut. S. III berichtet über den betreffenden Beschluss.

3) Vgl. Erläut. S. 33 f. mit Mells Aufsatz in den Mitt. des Instituts 21, 385 ff., insbesondere die beiden Stammbäume. 
einer Stelle kommt diese Anschauung noch zum Ausdrnck 1 ). Man wird das Festhalten an dem früheren Standpunkt bei einem Forscher, der mit den lokalen Fragen sich schon seit so vielen Jahrzehnten verdienstlich beschäftigt hat, begreifen und achten, aber doch hervorheben müssen, dass der Wert der Erläuterungen und die Hoffnung des ganzen Atlasunternehmens durchaus nicht in unsicheren Kombinationen über die Zeiten des 9., 10. und 11. Jahrhunderts, sondern in dem sicheren Tatsachenmaterial gelegen ist, das vom 13. Jahrhundert beginnt und gegen die Neuzeit her immer reichlicher strömt.

Damit soll freilich nicht gesagt sein, dass der historische Atlas für das frühere Mittelalter gar keine Früchte tragen könne. Schon was bisher an "Abhandlungen" vorliegt, würde eine solche Auffassung wiederlegen, denn von einem Aufsatze abgesehen (s. oben S. $562 \mathrm{f}$. Anm. 2, III.), nehmen mittelalterliche Fragen überall einen breiten und teilweise den ganzen Raum ein. Was in diesem einen Aufsatz Richter darlegt, ist allerdings von besonderem, methodischen Interesse; er gehört zu jener Gruppe von Hilfsarbeiten, welche die Verwendbarkeit moderner Gemeindegrenzen für den gesehichtlichen Atlas erörtern²). Diese Gemeindegreuzen könnten ja für die kartographi-

1) Inbezug auf Windberg und Mühlland meinte Strnadt Erläut. S. 12, ев веi möglich , die Ursprünge späterer Zustände bis auf die einzelnen Komitate zurückzuverfolgen ; dem sollte wohl der Stammbaum S. 14 entsprechen, welcher mit dem, comitatus Adalberonis a fonte fluminis Ilzisa usque ad fontem fluvii Rotala 1010" anhebt. Aber abgesehen davon, dass diese Ortsbezeichnungen in der Urkunde (DH. II. 217) nicht, so wie man bei dieser Wiedergabe glauben möchte, Grafschaftsgrenzen, sondern Grenzen eines Waldgebiets bedeuten, hat Strnadt selbst später, Archiv. f. öst. Gesch. 94, 278, die Urkunde verwerfend, auch die Existenz der Grafschaft Adalberts in Frage gestellt. An anderen Punkten liegt ein solcher Widerruf von Strnadt nicht vor, ja er wiederholt neuestens (Archiv 99, S. 10), , die bei Bearbeitung des hist. Atlas in Anwendung gebrachte Methode der Rekonstruktion " habe es ermöglicht, aus den kleineren und kieinsten Landgerichten der späteren Zeit die alten grossen zusammenzusetzen und dann aus letzteren die einzelnen Grafschaftsgebiete zu gewinnen*. Es muss gegenüber diesen sicher vorgetragenen Ansichten betont werden, dass ein bestimmter Nachweis, wornach ein einzelner Komitat sich genau mit einem der späteren Gerichte oder mit der Summe mehrerer solcher junger Bildungen decken würde, auch auf oberösterreichischem Gebiet nirgends erbracht ist. Die Ansicht, dass ein ein. zelnes Gericht der späteren Zeit ehemals cin Zentgericht oder eine Hundertschaft gebildet hätte (Erläut. S. 14, 1. Sp. unten, Archiv 91, 590 u. allgemein wiederholt Arch. 99, 10 f.), muss nach den Ausführungen Voltelinis (Arch. 94 S. $6 \mathrm{f}$.) wohl aufgegeben werden.

2) Für die österr. Länder wurde dasselbe Problem auch erörtert oder gestreift von Richter in den Mitt. d. Inst. 6. Ergbd., 865 ff. u. Deutsche Geschichtsbl. 4, 147 f.; von Grund, Mitt. des Inst. 2?, 186 und in der dort angezeigten Schrift 
sche Fixierung der Landgerichtsgrenzen in Fällen, wo keinerlei oder keine ins Einzelne gehenden Beschreibungen des Grenzverlaufs rorliegen, einen willkommenen Ersatz bieten; denn die Namen der Ortschaften, die zu diesem oder jenem Gericht gehörten, lassen sich häufig mit solcher Sicherheit feststellen, dass es nur darauf ankäme die alten Gemarkungen dieser Ortschaften zn finden, um zu den Gerichtsgrenzen $\mathrm{zu}$ gelangen. Dabei ist jedoch $\mathrm{zu}$ beachten, dass diese alten Gemarkungen nirgends direkt überliefert, sondern nur durch ihre etwaige Konservierung in jüngeren Grenzen, besonders in den Steuergemeindegrenzen erhalten sein können und dass auch deren Ursprung sich fast von Land zu Land anders gestaltet hat. Während in Niederösterreich bei der Bildung der Steuergemeinden zur Zeit des Kaisers Franz (1818 und in den folgenden Jahren) direkt an die damals bestandenen Gemeinden angeknüpft werden sollte, die freilich durch Eingemeindung der bisher ausserhalb der Gemeindeeinteilung gestandenen Gebiete mannigfache Änderungen erfahren konnten, ist in Steiermark bei der Bildung der Kreis-, Bezirks- und Gemeindeeinteilung von den Pfarrgrenzen ausgegangen worden: die Steuergeneindegrenzeu sind also hier für die Umgrenzung der alten Gerichte wertlos, dort unter gewissen Voraussetzungen verwertbar. Günstig gestaltet sich, wie es scheint, das Verhältnis für Kärnten, wo die neueren Einteilungen auf der alten Gerichtseinteilung aufgebaut sind, sowie für Görz und Gradiska, wo Gerichtseinteilung und Gemeindeeinteilung seit Alters in engem Zusammenhang stehen. Für Salzburg, wo der Josefinische Kataster nicht bestand, weil das Land zu jener Zeit noch nicht unter österreichischer Herrschaft gestanden hatte, war die Arbeit, als man 1828 die Katastrierung in Angriff nahm, schwierig, da es hier ältere Steuergemeinden nicht gab und die zur Zeit der bairischen Verwaltung gebildeten Steuerdistrikte als eine allzu willkürliche Schöpfung sich nicht zur Grundlage eigneten. Nach einigen Einwendungen der Linzer Regierung wusste es der Leiter der Arbeit im damaligen ,Salzachkreis“, Oberleutnant Gampert, durchzusetzen, dass die Steuergemeinden durch jeweilige Konzentrierung einer geeigneten Anzahl von Ortschaften und nachträgliche Vermessung des so gebildeten Körpers formiert wurden. Wie sich diese alten Ortschaften (Rüget,

Starzers; von Giannoni in den Blättern des Vereins f. Landeskunde von Niederösterreich 33, $475 \mathrm{ff}$. und in den Vierteljahrsheften für den geogr. Unterricht 1, $17 \mathrm{ff}$. (hauptsächlich mit Bezug auf Niederösterreich); ron Mell in den Deutschen Geschichtsblättern 6, $58 \mathrm{ff}$. (betreffend Kärnten, Görz u. Gradiska); von Pirchegger in den Erläut. z. hist. Atlas S. 30 (betr. Steiermark). Für Tirol werden die Bemerkungen von Wopfner, Das Almendregal S. 18 zil beachten sein. 
Rotten, Zechen, Kreuztrachten oder Viertel) auf die Pfleggerichte verteilten, ist uns zur Genüge, u. a. auch durch die Darstellung Kleimayrns ${ }^{1}$, bekannt. Man muss also, da bei der "Konzentrierung" dieser Einheiten nicht über die Gerichtsgrenzen hinübergegriffen wurde, annehmen, dass die Aussengrenzen der innerhalb eines Gerichts $1828 \mathrm{ff}$. gebildeten Steuergemeinden, mit den damals bestandenen Gerichtsgrenzen identisch sind. Das bildet für die Atlasarbeit eine wesentliche Erleichterung, da die Steuergemeindegrenzen genau vermessen und durch die bald darnach ausgegebene Übersichtskarte uns zugänglich gemacht wurden. Bei der Wichtigkeit, die also den Steuergemeindegrenzen hier zukommt, ist es sehr zu begrüssen, dass Richter den einschlägigen amtlichen Schriftenwechsel des Jahres 1828 ausführlich mitteilt. Seine Veröffentlichung wird auch für andere Länder ein gutes Muster abgeben. Im Hinblick auf die Fortsetzung dieser für den Atlas so bedeutenden Studien drängt sich dabei allerdings der Wunsch auf, dass Richter dem Leser, gewissermassen als Probe auf die angestellte Rechnung, auch überzeugende Beispiele vorgeführt haben möchte, in denen alte Gerichtsgrenzbeschreibungen von den ans den Karten ersichtlichen Steuergemeindegrenzen bestätigt werden, und dass er ihm klare Auskunft darüber gegeben hätte, ob diese Übereinstimmung sich in allen Fällen, wo bis 1828 keine Veränderung der Gerichtsgrenzen anderweitig bezeugt ist, wirklich bewährt2). Aber auch, wenn diese Hilfsstudien, vom Standpunkt des Kartographen gesehen, mit negativem Resultat enden und für das Alter der Gemeindegrenzen sich kein zwingender Beweis erbringen lässt, werden sie dem Historiker als Beitrag zur Kenntnis der staatlichen Reformtätigkeit des 18. und 19. Jahrhunderts wertvoll sein.

Von den übrigen "Abhandlungen", welche vorwiegend der mittelalterlichen Gerichtsentwicklung ihr Augenmerk zuwenden, verdient diejenige von Voltelini, die den 94. Band des Archivs einleitet, (oben S. 562, Anm. 2, I), die weiteste Beachtung. Sie ist aus dem Vortrag hervorgegangen, den V. auf dem Historikertag in Salzburg hielt, und sie eignet sich vorzüglich dazu die Abhandlungen zu eröffnen ${ }^{3}$ ). Die

1) Nachrichten rom Zustande der Gegend und Stadt Juvavia (1784) S. 419 ff.

2) Die allgemeine Bemerkung in den Erläut. S. 1, wornach ausser den Grenzbeschreibungen auch die Steuergemeindegrenzen, vielfach mit grossem Erfolg zur Grenzbestimmung verwendet werden konnten «, genügt doch nicht ganz; vielleicht mag Richter auch in dieser Hinsicht noch genauere Nachweise zu geben beabsichtigt haben, aber durch Erkrankung und Tod daran verhindert worden sein.

3) Die von Mell geäusserte Absicht, dem Band eine noch weiter ausgreifende Darstellang voranzustellen, welche die ganze Geschichte der hohen und niederen 
Frage nach der Entstehung der Landgerichte ist ja für einen historischen Atlas, der sich die Landgerichtskarte zur ersten Aufgabe macht, von ausserordentlicher Bedeutung, und die Art, wie V. sie behandelt, ist methodisch lehrreich für das ganze Unternehmen. Richter hatte die Vorstellung von der grossen Stabilität der Gerichtsgrenzen dem Atlas zu Grunde gelegt; Voltelini stellt sich nicht gerade in Gegensatz dazu, wohl aber bietet er eine höchst notwendige Ergänzung dieses Gedankens und ein unerlässliches Gegengewicht für die Ausführung. $\mathrm{Er}$ schildert uns, wie die Gerichtssprengel weit weniger stabil sind als die Pfarrbezirke; sie "schwanken vielfach, entstehen und vergehen und sind erst im Beginne der Neuzeit einigermassen feststehend geworden." Sind auch diese Worte (S. $11 \mathrm{f}$.) zunächst in Bezug auf Südtirol ausgesprochen, Voltelinis bevorzugtes Forschungsgebiet, das er auch für den historischen Altas zu bearbeiten auf sich nahm, so gewinuen sie in Rahmen seines Aufsatzes doch eine weit reichende Bedeutung. Denn indem V. daran geht, die Ursachen aufzuhellen, welche solche Schwankungen verursachten, greift er über Südtirol hinaus und überzeugt den Leser, dass dieselben Kräfte auch in den Nachbarländern gewirkt und ähnlichen Einfluss auf die wechselnde Gestalt der Gerichtseinteilung geübt haben können. Sehr daukenswert sind seine Ausführungen über die Wirkung zunehmender Besiedlung; sie müssen gerade für die Alpengegenden überall ernstlichst berücksichtigt und als eine Warnung vor allzu kühner Anwendung der retrogressiven Methode beherzigt werden. Wesentlich neue und eigene Wahrnehmungen bieten dann die Abschnitte über den Einfluss der Burgenverfassung auf die Bildung neuer Gerichte. Vielleicht kann man bedauern, da $B$ dem Verf. dabei die zahlreichen Einzelerscheinungen dieser Art aus Steiermark und Oberösterreich, die jetzt der 1. Atlaslieferung zu entnehmen sind, noch nicht zu Gebote standen. Um so mehr werden seine Ausführungen den Ausgangspunkt und die Richtlinie für weitere Forschungen zu bilden haben $\mathbf{1}$ ).

Die andere den Atlasabhandlungen eingereihte Arbeit Vol telinis (oben S. 562 f. Anm 2,V) behandelt ausschließlich südtirolische Verhältnisse, die Immunitäten des Hochstiftes Trient, der beiden Domkapitel

Strafgerichtsbarkeiten von ihren Anfängen bis zu ihrem, Aufhören", also wohl bis zu den Neuorganisationen des 19. Jahrhts., führen sollte (D. Geschichtsblätter 6; 64), hat sich anscheinend als nicht durchführbar erwiesen.

1) Dabei mag es sich vielleicht empfehlen auch das geographische Moment, die Lage der.Burgen, die als Gerichtssitze nachweisbar sind, genau zu beachten. Sie finden sich küineswegs.immer .inmitten ihres Gerichtsbezirkes, sondern häufig hart an der Grenze, als ob es gälte, die dahinter liegende Landschaft gegen Über- 
von Trient und Verona (welch letzteres in Judikarien einen geschlossenen Gerichtsbezirk besass), des Chorherrenstifts Au und des Klosters S. Michael an der Etsch, dann die grund- und leibherrliche Gerichtsbarkeit der Grundbesitzer. Auch hier sind Erörterungen von allgemeinem Interesse reichlich in die lokalen Einzeluntersuchungen eingestreut1). Im Zusammenhang der Atlasunternehmung erweckt aber auch die kartographische Frage Interesse, inwiefern die ron Voltelini geschilderten Verhältnisse auf der Karte dereinst zum Ausdruck zu bringeu sein werden. Für Burgfriede und auch für Herrschaftsitze und Schlösser, also für die verschiedenen Mittelpunkte einer oftmals geographisch nicht zu erfassenden niederen Gerichtsbarkeit siud Signaturen vorhanden. Wie aber werden Immunitäten, deren Herr die hohe Gerichtsbarkeit durch lange Zeiträume besass und sie dann wieder, etwa schon zu Ende des 13. Jahrhunderts, einbüsste, behandelt werden? Die durch ein Weistum von 1238 gegebenen Grenzen der drei judikarischen Dörfer, über die das Veroneser Kapitel herrsehte ${ }^{2}$ ), dürtten wohl als Landgerichtsgrenzen einzutragen sein, wie es ja auch sonst mit derartigen noch vor dem Endtermin der Kartendarstellung aufgehobenen Grenzen der hohen Gerichtsbarkeit zumeist gehalten worden ist $^{3}$ ). Schwieriger vielleicht wird die andere Frage sein, ob auch die Dekanien und Gastaldien, von denen Voltelini nachweist, dass sie eine wirtschaftliche, der Villenverfassung entsprechende Einteilung darstellen, in der Karte zum Ausdruck kommen sollen. Sie gänzlich beiseite zu lassen, wird deshalb kaum angehen, weil die Gastaldien doch unter Umständeu, gestützt durch die Bedeutung einer Burg, auf die Bildung der Gerichtssprengel einwirkten ${ }^{4}$ ) und weil eine besondere

griffe des Nachbarn zu schützen. So auf Bl. 9 Hüttenstein, Wartenfels von salzburgischer, Wildeneck von bairischer Seite. Haben da unzweitelhaft militärische Rücksichten eingewirkt, so wird solche exzentrische Lage des Gerichtssitzes bei Gerichten, die uns nur als unter einem Herren vereint entgegentreten, doch auch Schlüsse auf Selbständigkeitsbestrebungen u. Gegensätze in älterer Zeit zulassen.

1) Rietschel hat in der Ztschr. der Savignystiftung, N. F. Germanist. Abtlg. 28, $515 \mathrm{ff}$. als rechtsgeschichtlich besonders lebrreich die Abschnitte über Twing und Bann und über dje soziale Gliederung der Bevölkerung herausgehoben (Arch. 94, 404 ff.); vom Standpunkt der Diplomatik sei auf die neugewonnene Beurteilung der falschen Berengarurkunde für das Domstift Verona (Schiaparelli Nr. 113) und die zusammenhängende Besprechung dieser ganzen Gruppe von Immunitäts. urkunden ·(Arch. 94, $317 \mathrm{ff}$ ) hingewiesen.

2) S. Archiv 94, 327; über andere vielleicht auch darstellbare Immunitätsbezirke s. S. 396,398 .

s) Einige Ungleichmässigkeiten in der kartographischen Darstellung solcher Bezirke erwähnt Sieger in den Mitt. der geogr. Gesellsch. 1907, 251.

4) S. Voltelinis Bemerkungen S. 366 und 368 . 
kartographische Darstellung vom wirtschaftsgeschichtlichen Gesichtspunkt nicht so baì eintreten wird. Vielleicht ermöglicht die Schaffung neuer Signaturen für die betreffenden Atlasblätter einen Ausweg.

Es ist kein Unglück, dass Voltelini diese praktischen Fragen der Zukunft rorbehalten hat; nachdem iüber die rechtsgeschichtliche Entwicklung Klarheit gewonnen ist, werden sie von ihm gewiss auch in solcher Form gelöst werden, die dem geographischen Bedürfnis und der Einheit des ganzen Atlaswerkes Rechnung trägt. Seine Abhandlungen sind eben vorbereitende Schritte, während Richter und Strnadt die ihren erst nach Vollendung der Kartenblätter schrieben oder wenigstens veröffentlichten. In diesen treten daher die geographischen Gesichtspunkte stärker heraus, Richter wirft in der zweiten Abhandlung des Bandes (oben S. 562 f. Anm. 2, II) von neuem eine Frage auf, die er schon in seinen Untersuchungen zur historischen Geographie des Hochstifts Salzburg behandelt hatte und die dann auch von mir, als ich mit den Diplomen Ottos III. beschäftigt war, vor zwanzig Jahren berührt und in einem Punkte gefördert worden ist ${ }^{1}$ ): die Frage, ob es bei der Herstellung der grossen Besitzbestätigungen für Salzburg, welche mit der gefälschten Arnolfurkunde (Reg.. ${ }^{2}$ 1850) und zwei echten Urkunden Ottos II. anheben, beabsichtigt gewesen sei, dem Erzstift in gewissen Gebieten einen Ausschluss gräflicher Gerichtsbarkeit, also ein geschlossenes Immunitätgebiet zu sichern. Richters Haltung in dieser Frage ist im Laufe der. Jahre zurückhaltender geworden, ohne dass er schliesslich zur völligen Verwerfung seiner früheren Ansicht gelangen möchte. Vielleicht ist uns die ganz befriedigende Lösung durch Richters Krankheit und seinen allzufrühen Tod vorenthalten worden ${ }^{2}$ ) immerhin darf man seine Untersuchung als einen bedeutenden Fortschritt begrüssen; die übersichtliche Nebeneinauderstellung der betreffenden Teile von DO. II. 165 mit den älteren Rechtstiteln der Kirche fördert den Gegenstand ebensosehr wie die Bezugnahme auf die durch Seeliger hervorgerufene neuere Literatur über das Wesen der Immunität ${ }^{3}$ ).

1) Mitt. des Inst. 10,607 ff.

2) Die traurige Anmerkung der letzten Seite (S. 62), in der Richter erklärt, dass er leider krankheitshalber auf die weitere Ausführung dieser interessanten Frage verzichten müsse, ist rom 31. Jänner 1905 datiert, also sechs Tage vor seinem 'Tode geschrieben!

3) Richters Abhandlung legt mir nahe, einige weitere Bemerkungen über die salzburgischen Konfirmationen vorzubringen, die ich aber, da sie hier den Rahmen der Besprechung sprengen würden, an anderer Stelle veröffentliche. 
Eine deutliche geographische Gliederung weisen die Abhandlungen auf, welche Strnadt im Anschluss an den historischen Atlas veröffentlicht (oben S. 562 f. Anm. 2, IV, VI und VII) und die an Umfang weitaus über die besprochenen Arbeiten von Richter und Voltelini hinausgehen. Es ist Strnadts Plan, jedem der vier Bestandteile des Landes ob der Enns, die im Jahre 1779 als Landesviertel bezeichnet wurden und die in der Tat durch ihre geschichtliche Entwicklung geschieden sind, eine besondere Abhandlung zu widmen, welche den betreffenden kurzgefassten Abschnitt der Erläuterungen weiterführt. Dabei ist die Einrichtung von Strnadt bisher stets so getroffen worden, dass die letzten Kapitel jeder einzelnen Abhandlung sich am engsten mit den Erläuterungen berühren, indem sie von Gericht zu Gericht fortschreitend das dort Gesagte wiederholen und ergänzen. Diese Partien der Abhandlungen sind mit den Erläuterungen inhaltlich so sehr verwandt, dass beide Arbeiten stets nebeneinander zu beuützen sein werden ${ }^{1}$ ). Das ist allerdings eine etwas unbequeme Einrichtung und man kann sich manchmal des Eindrucks nicht erwehren, dass sich die Verteilung des Stoffes zwischen den Erläuterungen und den Ablandlungen praktischer und gleichmässiger hätte gestalten lassen. Indes sind dem Verf. diese letzten Kapitel jeder einzelnen der drei Abhandlungeu offenbar gar nicht die Hauptsache gewesen ${ }^{2}$ ); worauf er viel mehr Raum und Mühe verwendet hat und anscheinend auch mehr Gewicht legt, das sind die übrigen Abschnitte der Abhandlungen, in denen die ältere Geschichte jedes einzelnen Landesviertels nach bestimmten Gesichtspunkten untersucht und dargestellt, also gewissermassen die Verknüpfung der späteren Gerichtseinteilung mit den ältesten Zuständen des Landes angestrebt wird. Hier handelt Struadt über Gau- und Grafschaftseinteilung, über den Gang der Besiedlung, über die ersten welt-

1) Da in den Erläut. die zugehörigen Stellen der Abhandlungen, weil damals noch ungedruckt, nicht zitiert werden konnten, und da anderseits Strnadt in den Abh. zwar manchmal auf die Enläut. hinweist, aber zumeist ohne die Stelle genauer zu bezeichnen, werden folgende Anhaltspunkte nicht überflüssig sein: Zu Erläut. S. 9 f. vgl. Archiv 94, 293 bis 297; zu Erläut. 10 bis 12 vgl. Arch. 94, 282 bis 292 ; zu Erläut. 13 f. Arch. 94, 217 bis 252 ; zu Erläut. 14 untere Hälfte vgl. Arch. 94,583 bis 599 ; zu Elläut. 15 bis 18 einzelne Nachträge Arcì. 94, 599 bis 609 ; zu Erläut. 18 bis 22 Arch. 99, 214 bis 290; zu Erläut. S. 18, 2. Sp. unten letzte u. S. 19 erste Zeilen, 80wie zu Erläut. S. 23 Arch. 99, 1ذう bis 165 .

2) Aus Strnadts Vorwort zu seiner zweiten Abhandlung, Archiv 94, 467, gewinnt man fast den Eindruck, dass diese letzten Kapitel geschrieben seien, uil ,für Unterbringung der Grenzbeschreibungen zu sorgen * Über diese s. unten S. $587 \mathrm{ff}$. 
lichen und geistlichen Grundbesitzer, über die Entwicklung der Landesgrenze im Norden und Süden und über mancherlei neue und alte Fragen, die uns die Vorgeschichte des heutigen Oberösterreich aufgibt. Der Stoff ist so mannigfach, dass es unmöglich wäre, darüber im einzelnen zu berichten. Nur einige Punkte seien aus der leider durchaus nicht glücklich geordneten Masse ${ }^{1}$ ) hervorgehoben. Den vom 16. Juni 1361 datierten Lehenrevers der Grafen von Schaumberg, dessen Angaben über die Gerichtsverhältnisse mehreres Auffallende in sich schliessen, hat Strnadt eingehend untersucht (Arch. 99, 191 bis 204) und er ist auf Grund innerer und äusserer Merkmale (bei den letzteren freilich nur auf das Urteil anderer sich stützend) zu der Ansicht gelangt, dass diese Urkunde unmöglich echt sein könne. Er hält sie für das Erzeugnis eines Mannes aus der Kanzlei der Grafen von Görz, welcher, vorübergehend im Auftrag seiner Herren am österreichischen Hofe weilend, von Herzog Rudolf IV, zur Herstellung dieser Fälschung verwendet worden sei: gewiss eine sehr kühne Annahme²), aber im Zusammenhang mit der sonstigen Fälschertätigkeit an Rudolfs Hof jedenfalls zu beachten. Die weitausgesponnenen Erörterungen über Heimat und Reihenfolge der Traungauer Otakare (Arch. 94, 510 bis 582) geben Anlass zur Mitteilung eines hübschen

1) Die am Schluss jeder Abhandlung beigegebenen Inhaltsubersichten sind dankbar zu begrüssen, auch das Programm, das Strnadt Archiv 94 S. 86 seiner ersten Abhandlung vorausschickt; aber sie beheben nicht die Mängel der Disposition, die sich besonders in der letzten Arbeit (Arch. 99) empfindlich fühlbar machen. Da wird zuerst das Thema der Gaue und Gratschaften angeschlagen (S. 7 bis 13), dann auf Grundbesitzverteilung im Atergau übergegangen (S. 14 bis 35, wobei zunächst ohne Überschrift Bamberg, dann zehn andere durch besondere Titel hervorgehobene Grundbesitzer folgen), endlich S. 35 die Frage der Standesverhältnisse in Angriff genommen; aber dieser Gegenstand wird nachträglich wieder durch Einschiebungen über die Grafen im Atergau (S. 49 bis 56) und über Besitzverhältnisse im nördlichen Teil des Hausruckviertels (wobei die fettgedruckte und in die Übersicht aufgenommene Überschrift ,Zwischen Donau, Rotensala \& u.s. w., S. 64, den Leser nicht ahnen lässt, welches der drei Themata er hier finden wird) unterbrochen, dann S. 72 ohne jede Überschrift wieder autgenommen u. s. w. Dabei ereignet sich, dass S. 90 ff. auch ein ,Blick über die Donau « geworfen, also das Hauptgerippe für die Stoffanordnung der Abhandlungen umgestossen wird und dass S. 88 ff. eine Anmerkung so lange wird, dass sie im Text gedruckt werden und mit neuen Anmerkungen versehen werden muss. Von S. 109 beginnen Zusammenfassungen und Rückblicke, zwischen hinein aber immer noch Nachträge zu den früberen Themata, anderseits S. 155 bis 165, als Vorläufer der auf die Landgerichte bzgl. Schlussabschnitte (S. 214 ff., s. vorige Seite), Ausführungen über die Grenzverhältnisse in der Niederen Kessla.

2) Strnadt sieht selbst, Arch. 99, 4, voraus, dass er mit seiner Erklärung .auf Widerstand stossen werde. 
Gutachtens von Bloch über die Schrift der angeblichen Schenkungsurkunde des Markgrafen Ernst für Melk ${ }^{1}$ ), zu eingehenden, auch die Urbare späterer Zeit heranziehenden Untersuchungen über die im Chiemgau und Sundergau gelegenen Güter, welche im Jahre 959 von Otto I. dem Domstift von Salzburg und dem Kloster S. Emmeram zu Regensburg bestätigt wurden (DDO. I. 202, 203), zu einer Beschreibung des im 14. Bande der Monumenta Boica mangelhaft abgedruckten, 1281 nach älterer Vorlage hergestellten Traditionsbuchs von Geisenfeld, endlich zur Besprechung eines D. Heinrichs V. für S. Georgen im Schwarzwald (Stumpf Reg. 3026), in welchem neben anderen Markgrafen auch ein "Odachor" als Intervenient genannt ist. Wird nun auch die in diesem Fall versuchte Verwandlung des Odachor in Tietbald (!), den Markgrafen von Vohburg, und das Bestreben, der Kanzlei Friedrichs II. zuzumuten, dass sie von den sardinischen Beziehungen dieses Kaisers irregeleitet, bei der Transumierung des verlorenen Originals eine so unglaubliche Entstellung des Namens begangen hätte, kaum eine ernste Würdigung finden ${ }^{2}$ ), so zeigt sich doch überali ein gewisser Lifer des Verfassers deı Dingen auf den Grund zu sehen. Gelungen ist ihm dies freilich nicht überall. Einen argen Missgriff begeht Strnadt bei Beurteilung derjenigen Urkunde Heinrichs II, welche dem Kloster Niedernburg zu Passau einen sehr bedeutenden Anteil am Nordwald zuspricht, das ganze Gebiet am linken Donauufer von der bei Passau mündenden Ilz abwärts bis zur Rodl, die oberhalb Linz in die Donau fällt. Bresslau hat das auf uns gekommene Stück (DH. II. 217) für eine zu Ende des 11. oder Anfang des 12. Jahrhunderts angefertigte Nachzeichnung erklärt; aber er ist, indem er schon dem Regest beifügt, dass die angegebenen Grenzen des geschenkten Gebietes unverbürgt seien, dennoch für die Benützung einer echten, uns nicht mehr erhaltenen Vorlage eingetreten und zwar deshalb, weil sich an deu Schriftzügen der Nachzeichnung und am Diktat der Einfluss eines ganz bestimmten Schreibers erkennen lässt, der in Beziehungen zu Abt Godehard gestanden haben und der von 1009 angefangen durch mehrere Jahre tätig gewesen sein muss. Strnadt behandelt dieses Stück als Fälschung ${ }^{3}$ ). Wenn er meint, es sei überhaupt keine Waldschenkung an Niedernburg erfolgt oder dieselbe hat sich — höch-

1) Vgl. über dieses Stück jetzt die treffenden Worte von Mitis, Studien zur. älteren österr. Urkundenwesen $215 \mathrm{ff}$.

2) Über St. 3026 vgl. indes auch Hirsch in den Mitt. des Inst. 7. Ergbd. $487 \mathrm{ff}$; dann auch den von Strnadt im Arch. $99 \mathrm{~S} .5$ mitgeteilten, aber in seiner Bedeutung doch wohl unterschätzten Einwand Bresslaus.

3) Arch. 94, $276 \mathrm{ff}$, vgl. auch S. $659 \mathrm{f}$. 
stens auf den schmalen Waldstrich am jinken Ilzufer erstreckt", so wäre das letztere allenfalls noch mit Bresslaus Urteil vereinbar. Aber Strnadt geht weiter und sucht eine Erklärung zu geben. „Die Fälschung" so meint er "erfolgte im bequemen Anschlusse an die beiden echten Schenkungen Heinrichs II. an Niedernburg 1010, 19. April besonders an letztere". Man könnte nun zugeben, daß der Name des Grafen Adalbert dem DH. II. 215 für Niedernburg entnommen wäre; aber wahrscheinlich ist dies keineswegs, denn den grössten Teil des Kontexts und auch gewisse Protokoll-Eigentümlichkeiten kann der Fälscher unmöglich den erhaltenen Diplomen für Niedernburg, er muss sie vielmehr einem verlorenen, echten, von dem Godehardschreiber verfassten Diplom entnommen haben ${ }^{1}$ ). Und auch dieses verlorene Original enthielt eine Waldschenkung und gab die Grenzen dieses Waldes an, denn auch die den Wald betreffenden Worte schliessen deutliche Spuren von dem Diktat des Godehardschreibers in sich ${ }^{2}$ ). Diese für die Beurteilung von DH. II. 217 so wesentlichen Umstände, welche Bresslau zwar nicht im Einzelnen angeführt, auf die er aber durch sein Urteil über Schrift und Diktat den Sachkundigen genügend aufmerksam gemacht hatte, sind ron Strnadt unbeachtet geblieben, weil er mit der diplomatischen Methode und schliesslich auch mit der Art, die Diplomata-Ausgabe zu handhaben, nicht genug vertraut ist. Er hat, Bresslaus Hinweis auf ,D. 198“ missverstehend, anstatt der Nummer die Seite 198 des 3. Diplomatabandes aufgeschlagen und deshalb (Arch. 94, 276) eine der Bamberger Schenkungsurkunden Heinrichs II. vom 1. November 1007 als diktatverwandt mit DH. II. 217 angeführt ${ }^{3}$ ), ohne zu bemerken, dass sie gar keine Verwandtschaft mit

1) Der Eingang von DH. II. 217 bis, inibi deo famulantium * stimmt grossenteils wörtlich mit DH. II. 198, 229, der Schluss von, per hanc regalis precepti nostri paginam angefangen, wenn man von der Poen absieht, wörtlich mit DH. II. 229. Wollte man nun etwa annehmen, das letztgenannte Diplom selbst, obwohl in Niederaltaich liegend, habe um 1100 einem Passauer Fälscher als Vorlage gedient, so wäre nicht einzusehen, wieso dann das Eschatokoll von DH. II. 217 so wesentliche und richtige Abweichungen von DH. II. 229 zeigen könnte. A as den Niedernburger Diplomen von 1010 könnte das Eschatokoll auch nicht erklärt werden, da die Stellung des Monogramms und das, recognovi in XI deutlich auf den Godehardschreiber hinweisen.

2) $\mathrm{Zu}$ den Ausdrücken der Grenzbeschreibung: in longitudine', in latitudine vero', usque ad fluvium Danubii‘ vgl. DH. II. 229; zn ,portionem silvȩ vgl. ,partem silvę in DH. II. 231.

3) So wie Strnadt hier die Zitierweise Bresslaus missversteht, so bedient er sich auch selbst bei Anführung von Diplomen einer unpraktischen Seitenzitierung, die zu der merkwürdigsten Verwirrung führen könnte: ,Mon. Germ. Dipl. O. IIr, 148. (Arch. 94, 474) bedeutet bei ihm, was wir DH. II. 122 nennen; „Mon. Germ. 
dem zu untersuchenden Stück hat, ohne auch nur zu fragen, wieso denn Bresslau zu einem derartigen Urteil gekommen und wie eine solche Verwandtschaft bei der angeblichen Fälschung zu erklären sein sollte. Ist es nun aber sicher, dass DH. II. 217 auf ein verlorenes echtes. Original zurückgeht, das zum grössten Teil gleich lautete wie die Nachzeichnung, so bedarf auch alles das, was Strnadt über die Besiedlung der betreffenden Gegenden sagt, einer vorsichtigeren Fassung. Die Urbare des späten Mittelalters und besonders die des 16. Jahrhunderts gestatten zwar zu erkennen, dass ein grosser Teil des Besitzes zwischen Ilz und Rodl, also innerbalb des in DH. II. 217 begrenzten Raums, später in weltlichen Händen war; aber es ist doch nicht ausgeschlossen, dass diese ihn erst nach 1010 an sich brachten. Ein. zwingender Beweis gegen die Glaubwürdigkeit des Diploms kann also. aus diesen späteren Verhältnissen, welche Strnadt schon in seiner Erstlingsschrift ${ }^{1}$ ) und nun wieder eingehend dargestellt hat, doch wohl nicht gewonnen werden.

III.

Der "Rekonstruktion des Besitzstandes der weltlichen: Grundherrschaften im Mühllande zu Beginn des 13. Jahrhunderts" hat Strnadt auch eine besondere Karte gewidmet, die der ersten von seinen Abhandlungen beigegeben ist. Sie muss, im Zusammenhang mit der seiner letzten Abhandlung beigefügten „Kartenskizze über die Besitzverteilung gegen Ende des 12. Jahrhunderts", hier umsomehr erörtert werden, als es sich in beiden Fällen um eine Erweiterung des Altasprogramms handelt, über deren Zweckmässigkeit man verschiedener Meinung sein $\mathrm{kann}^{2}$ ). Das erstgenannte Blatt hält den Masstab der Landgerichtskarte $(1: 200.000)$ ein und ist sowie diese auf Grund der Generalkarte des k. u. k. militär-geographischen Institutes hergestellt; Terraindarstellung und Flussnetz stimmen also

D. O. III. 176, 188 " (Arch. 99, 13) bedeutet DH. II. 148, 158 u. s. f.; ausführlicher wird Arch. 94, 523 zitiert: „Mon. Germ. DO. I, 281 Nr. 202 und ,a. a. O. 282, Nr. 263*, wobei aber „263" hier und S. 530 in , $203^{\circ}$ zu verbessern ist; wenn im Arch. 94, 473 einmal ausnahmsweise nur nach der Nummer zitiert wird ,Mon. Germ. O. II, 165", womit nun wirklich DO. Il. 165 gemeint ist, so erklärt sich dies daraus, dass Strnadt das Zitat aus Richters Aufsatz herübernimmt.

1) ,Versuch einer Geschichte der passauischen Herschaft im oberen Mühlviertel namentlicn des Landgerichtes Velden " im Zwanzigsten Bericht über das. Museum Franzisco-Carolinum in Linz (1860), S. 73 bis 288.

2) Von der in $1: 500.000$ dargestellten Grafschaftskarte des Chiemganes, welche der zweiten Strnadt'schen Abhandlung beigegeben ist, soll, weil sie einen andern $\mathrm{Zweck}$ verfolgt, hier abgeșehen werden. 
mit den betreffenden Teilen der Atlasblätter $1^{\mathrm{a}}, 1^{\mathrm{b}}, 4$ und 5 überein, auch die Schrift ist mit Ausnahme der Gerichtsnamen zumeist dieselbe; der Unterschied ist nur, dass hier durch verschiedene Farben (teils durch Flächenkolorit, teils durch farbige Umrandung, zum Teil auch durch farbiges Unterstreichen der Ortsuamen) die Besitzverhältnisse angezeigt, dort durch Eintragung der Grenzen die Gerichtsbezirke gekennzeichnet sind. Diese enge Verwandtschaft der beiden Karten erscheint als ein erstrebenswerter Vorteil, sie ermöglicht die Vergleichung der Besitzverhältnisse mit der Gerichtseinteilung und sie könnte wohl den Wunsch rege werden lassen, eine derartige Doppeldarstellung auch für andere Gebiete zu besiłzen. Vermutlich waren es hauptsächlich finanzielle Gründe, welche in zweiten Fall ein Abgehen von diesem Massstab und dieser Methode, den Besitzstand zu veranschaulichen, herbeifübrten. Die Besitzverteilung im Hausruckviertel und den anstossenden Gebieten ist nämlich (was freilich auf der Karte selbst nicht angegeben ist) im Masse $1: 300.000$ gezeichnet; diese Karte entbehrt der Terraindarstellung, stimmt auch in dem Verlauf der Gewässer und in der Lage der Ortschaften nicht jmmer genau mit der Landgerichtskarte überein, so dass eine Vergleichung mit derselben nicht gut durchzuführen ist; sie bedient sich zur Bezeichnung der Besitzungen nicht der Farben sondern verschiedener anderer Mittel, roter Anfangsbuchstaben, welche, neben die Ortsnamen gesetzt, den Besitzer andeuten, und nusgeschriebener Besitzernamen, welche über diejenigen Gegenden, in denen der betreffende Besitz stark vertreten ist, sich hinziehen. So entsteht eine Karte, die weniger übersichtlich aber auch weniger ausdrncksfähig ist als die andere ${ }^{1}$ ); denn der Raum reicht in vielen Fälleu nicht aus, alle Orte einzutragen oder mit den entsprechenden Anfangsbuchstaben zu versehen, an denen sich Besitzungen dieser oder jener Art nachweisen lassen²). Die Karten-

1) Arch. 99, 4 sind derselben Kartenskizze noch weitere Aufgaben gestellt: sie soll zur Orientierung, über die räımliche Verteilung der Gemeinfreien dienen, zugleich ein ungefähres Bild der beiden Grafschaften im Hausruck und im Atergau mit Ausblicken über die Donau und über die Traun darbieten ": die Grafschaften sind durch römische Ziffern gekennzeichnet und ihre angenommenen Grenzen rot eingetragen.

2) So konnte die Lage des Rebgauer Besitzes wegen Raummangel nicht durch Beifïgung der Initialen zu den einzelnen Orten sondern nur durch den ausgeschriebenen Namen angedeutet werden; bei Seitenstetten musste Redlham. (Arch.99, 20), bei Kremsmünster Gallaberg (ebenda) wegbleiben; bei beiden wird die Lage indes im Text genügend gekennzeichnet. Auffallender ist, dass die Signaturen D. S. und E. S. (Domstift und Erzstift Salzburg) nur bei Puchkirchen, Ungenach, Ampflwang u. Köppach anzutreffen sind; wenn die im Arch. 99, 26 
skizze kann also keineswegs allen Besitz wiedergeben von dem die Quellen Zeugnis ablegen, sie erweist sich gegenüber dem Reichtum an Urkunden, den einzelne Kirchen bewahrt haben, unzulänglich. Auf der anderen Seite ist dieser Reichtum sehr ungleichmässig verteilt. Bei der Mehrzahl der Grundbestzer ist der Besitzstand ,gegen Ende des 12. Jahrhunderts" nicht auf Grund von Urkunden dieser älteren Zeit eingetragen, sondern mit Hilfe von jüngeren Güterverzeichnissen rekonstruiert. Für Seitenstetten und Kremsmünster sind es die um 1300 angelegten Urbare, das von Aspach gehört dem 14. Jahrhundert an, ebenso das grosse Schaumburger Urbar im Stiftsarchiv zu St. Florian ${ }^{1}$ ). Mehrere wichtige Grundbesitzkomplexe lassen sich aber nur mit Hilfe neuzeitlicher Verzeichnisse erkennen. Bei den Bambergischen Gütern bedient sich Strnadt (Arch. 99, 14) eines Urbars vou 1581, bei den Regauischen Eigen eines solchen von 1538 (ebda. 17); für die Besitzverhältnisse im Mühlviertel sind Urbare von Rannariedl, die 1510 und 1581, angelegt sind, dann das Falkensteiner Urbar von 1570 und ähnliche Quellen späten Ursprungs (Arch. 94, 253 ff.) von entscheidender Belleutung'2). Man wird nun gewiss nicht bestreiten können, dass

Anun. 2 aus dem Puchheimer Urbar angetührten Vogtgüter alle salzburgisch waren, so wären ausserdem noch einige andere Orte mit dem D. S. zu versehen gewesen. Auch zu Heft (Höft, Höftberg) bei Gaspoldshofen hätte (Arch. 99, 28 Anm. 1) diese Signatur gestellt werden können; dann entweder zu Streit bei Unterach oder zu Fornach (Arch. 99, 29 Anm. 2 und 36 Anm. 2; dass Strnadt von Hituthalers Ortserklärung da und dort abweicht, ist von ihm im allgemeinen S. 29 Anm. 5 vermerist, hätte aber besser wohl im einzelnen gesagt werden sollen; auf S. 29 fehlen auch zwischen Anm. 4 und 5 die Hinweise auf das Salzb. Urkundenbuch 1, 695 und 721). Auch der schon im 8. und wieder im 10. Jahrhundert bezeugte salzburgische Besitz zu Bachmanning und Niedertalheim (Salzb. Urkdb. 1 S. $5,20,42,44,70,99)$ ist auf der Karte nicht als solcher bezeichnet. Die Signatur für das Stift St. Peter (S. P.) steht bei Powang (an den Qucllen der Dürren Ager, wofür aber der Text Arch. 99, 30 ff. den Beleg, Salzb. Urkdb. 1, 31, nicht anführt), Breitenau und Lab (bei Wels, wo man im Text S. 31 neben einzelnen einschlägigen Traditionen, Salzb. Urkdb. 1, 317, 345, 396, 434, 448, auch das Urbar vom Ende des 12. Jahrh., ebenda 515, vermisst); St. Petrer Besitz gab es aber auch an andern Orten in Oberösterreich, so, wie schon aus Strnadts Text S. $30 \mathrm{f}$. ersichtlich, bei Pilsbach (Redlberg), Schwanenstadt, Otnang, Wolfseck, wo überall auf der Karte das S.P. fehlt, dann noch an weiteren gleichfalls in den Rahmen dieser Karte fallenden Örtlichkeiten, s. Salzb. Urkb. 1, 352, 433, 527,550 .

1) Von diesem ist auch in Strnadts älterer Abhandlung, Peuerbach ", im 27. Bericht über das Museum Eranzisco-Carolinum in Linz (1868) vielfach Ge. lrauch gemacht.

2) Die in den Wiener Archiven vorbancenen Urbarien hat jetzt, erst nach Abschluss der besprochenen Abhandlungen zum Atlas, Kaser im Auftrag der 
auch solche Quellen unter Umständen sehr wertrollen Aufschluss über die Besitzverhältnisse in viel älterer Zeit gewähren können. Aber die grosse Verschiedenheit der uns vorliegenden Quellen nach' Alter und Beschaffenheit muss doch ernstlich erwogen werden, wenn ein Bild der Besitzverteilung für eine bestimmte Zeit entworfen werden soll. Ich befürchte, dass Strnadt diese Quellenlage allzu optimistisch angesehen und sich schon im Text, sowohl inbezug auf die Verhältuisse nördlich der Donau als auch für Atergau und Hausruck, viel zuversichtlicher über die Besitzverhältnisse geäussert hat, als es die Quellen rechtfertigen ${ }^{1}$ ). Unbedingt aber halte ich die kartographische Fixierung der gewonnenen Schlüsse für ein gefährliches Experiment, weil das Kartenbild dem Benützer anscheinend gesicherte Ergebnisse bietet nnd bieten muss, auch wo die Quellen hiefür nicht ausreichen. Es gehörte zu den besten und fruchtbarsten Gedanken Richters, dass er sich gegen die kartographische Fixierung der hofrechtlichen oder grundherrlichen Beziehungen aussprach. "Dienste und Pflichten" so sagte er in seinem Programm für den historischen Altas der österr. Alpenländer „lasten bei uns auf den einzelnen Höfen und die Zersplitterung geht so weit, dass eine Ausscheidung nach Flächen nicht mehr möglich ist"2). Er berief sich dabei auf den von ihm seibst angestellten Versuch, den Inhalt des ausführlichen Urbars, welches man im Jahre 1525 über den salzburgischen Besitz in Baiern angelegt hat, "kartographisch zu verwerten". Das Ergebnis sei wohl die Veranschaulichung grösserer oder geringerer Dichte der betreffenden Höfe gewesen ,aber ein territorialer Abschluss war nirgends zu gewinnen" und zwar deshalb, weil die Befugnjsse sich nicht auf zusammenhängende Flächen beziehen, die in den gewöhnlichen Karteumasstäben noch darstellbar sind". Beim Anblick der Besitzverteilungskarte für llzgau und Mühlland möchte man auf den ersten Blick glauben, was Richter für unmöglich erklärt, sei Strnadt doch gelungen,

Kais. Akademie der Wissenschaften verzeichnet (Sitzungsberichte der phil.hist. Kl. 161. Bd.); daraus werden sich vielleicht einige Nachträge zu dem von Strnadt benützten Materiale ergeben, so (S. 24) bei Falkenstein Urbare von 1537, 1554, bei Rannariedl (S. 26) eines von 1570 und eines mit der Aufschrift 1581, das Kaser als, wabrscheinlich von 1574 " bezeichnet.

1) Man vgl. Arch. 99, 16: , der günstige Quellenbestand gestattet die gesamte Grundverteilung " (diese Worte, zugleich als Überschrift dienend, sind von Strnadt fett gedruckt), festzulegen und auf dieser Grundlage zu völlig oder grossenteils gesicherten Folgerungen zu gelangen".

2) Richter, Über einen hist. Atlas (Sonderabdruck aus der Festgabe für Krones, 1895) S. 15, Mitt. der geogr. Gesellschaft 1896, 538 f., Korrespondenzblatt des Gesamtvereins 44 S. $77 \mathrm{f}$. 
denn hier sind trotz des gewöhnlichen Nasstabs grosse Flächen mit einheitlichem Flächenkolorit als geschlossener Besitz gekennzeichnet, andere, bë denen Strnadt solchen Zustand nicht für sicher aber für wahrscheinlich hält, mit farbiger Umrandung eingeschlossen. Sind diese Annahmen geschlossener Grundherrschaften aber auch wirklich berechtigt? Und wenn sie etwa da und dort fürs 16. Jahrhundert zutreffen, ist das für den Beginn des 13. Jahrhunderts auch so sicher, dass man wageu darf die Farbenstriche in so ruhigen Zügen in eine nach ibrer Überschrift diesem Zeitraum angepasste Karte einzutragen? Ich denke, der Umstand dass innerhalb des grünumrandeten Griesbachischen Besitzes um Rohrbach eine Reihe roter, Falkensteinischer Enklaven sichtbar wird und dass es solchen Streubesitz auch viel weiter westlich bei Wingersdorf, gleichfalls in dem grüneingeschlossenen Gebiet, gegeben zu haben scheint'1), sollte doch vorsichtiger machen. Sehr auffallend ist auch, dass Strnadt „das Gebiet der Kirche Passau" gar nicht farbig bezeichnet hat, wie er sagt ,um dasselbe gegen die fremden Grundherrschaften besser sich abheben zu lassen". Aber dies kann doch nicht der einzige und der durchschlagende Grund für eine so wichtige Entscheidung gewesen sein, die gerade den Hauptzweck der Karte - Vergleich des weltlichen Besitzes mit dem geistlichen - vereitelt, da nun niemand ersieht, wo denn passauische Güter lagen²). Der Grund muss vielmehr der gewesen sein, dass sich der passauisehe Besitz nicht mit genügender Sicherheit feststellen und eintragen liess. Ist dem so, dann liegt es nahe auch hier die Gedanken anzuwenden, die Richter in Bezug auf die Eintragung ron Ortschaften zur Veranschaulichung der Besiedlung schon vor langem treffend aussprach ${ }^{3}$ ). Sowie in diesem Fall „die Eintragung der Namen welche in einer gewissen Quellengruppe gerade zufällig überliefert sind, in eine Karte welche einen allgemeinen Titel führt" nach Richters Worten „streng genommen unlogisch ist und leicht zu falschen Vorstellungen führen kann", so halte ich es für eine nicht. unbedenkliche Abweichung vou der richtigen Methode, eine kartographische Darstellung des Gesamtbesitzstands oder auch nur des gesamten weltlichen Besitzstands einer Gegend zu versuchen, wenn nicht für alle daran beteiligten Grundherrschaften ein gleich ausführliches, gleich

1) Archiv 94, 276, wo Strnadt auch Aufklärungen über Zweck und Bedeutung dieser Karte gibt.

2) Denn dass nicht alles Unkolorierte auf der Karte, die sich von Passau bis Linz erstreckt und weit nach Böhmen hineinreicht, als passiluisch zu betrachten sein kann, leuchtet jedem ein.

3) Mitt. des Instituts 1. Ergbd. 592; vgl, auch 5. Ergbd. 66. 
altes und gleich zuverlässiges Quellenmaterial vorliegt. „Ganz anders gestaltet sich “, um mit Richter fortzufahren, „die Sache, wenn die Karte selbst nur zur Erläuterung“ einer bestimmten Quelle oder Quellengruppe, in unserem Fall also, wenn sie zur Erläuterung bestinumter Urbare "dienen soll". Sie wird dann "nicht mit der Prätension einer erschöpfenden Darstellung “ auftreten, sie wird „als Quellenbearbeitung; nicht als Darstellungsversuch sich zu geben haben. ${ }^{*}$

IV.

Man mag indes über die Durchführbarkeit und den Wert solcher Besitzverteilungskarten verschiedener Meinung sein, das ist doch gewiss, dass sie mit den Zielen, die dem historischen Altas in erster Linie gesteckt sind, also mit der Landgerichtskarte nicht so unmittelbar zusammenhängen, wie alles das, was über den Bestand und das gegenseitige Verhältnis der Gerichtsbezirke Aufschluss gewährt. Aus der Zahl der Quellen, die für diese Fragen in Betracht kommen, ragt nun eine Quellengattung am meisten hervor, die bisher nicht selrr beachtet wurde, durch die Vorarbeiten zum Atlas aber zu Ehren gekommen ist; ich meine die Grenzbeschreibungen der Gerichte, deren es einige schon aus älterer Zeit, eine zunehmende Masse aber seit dem 15. und 16. Jahrhundert gibt. Sie sind die wichtigste Grundlage für den Aufbau der Landgerichtskarte, auf ihnen beruht nicht nur der Text der Erläuterungen und der Abhandlungen sondern auch die Karte selbst. Bei dieser Lage habeu es die Bearbeiter durchwegs für ihre Pflicht gehalten, die von ihnen benützten Grenzbeschreibungen auch anzuführen und teilweise mitzuteilen, um dem Benützer Rechenschaft. zu geben und eine Nachprüfung zu ermöglichen. Aber sie sind in diesem Punkt nicht in ganz gleichmässiger Weise verfahren, wie denn vom Anfang an in den hierüber abgegebenen Äusserungen verschiedene Ansichten zum Ausdruck kamen. Zuerst hatten Mell und Kapper den Abdruck der Grenzbeschreibungen gefordert1), dann erinnerte Richter, obne zur Frage des Abdrucks Stellung zu nehmen, daran, dass auch für die Grenzbeschreibungen die Erhaltung und Aufbewahrung sich je nach den urchivalischen Verhältnissen der Kronländer sehr verschieden gestalten ${ }^{2}$ ); ein Jahr später berichtete Mell, dass "seitens der Atlaskommission" bisher an Publikation der Grenzbeschreibung "nur insoweit gedacht" worden sei, ,als man für eine solche die einzelnen Landespublikationsinstitute interessieren wollte",

1) Mitt. des Inst. 21, 392 und Deutsche Geschichtsblätter 2, 226.

2) Deutsche Geschichtsblätter 4, 146. 
und er deutete an, dass die Verwirklichung dieser Absicht bishin keine wesentlichen Fortschritte gemacht habe ${ }^{1}$ ). Schliesslich haben vach dem Erscheinen der ersten Lieferung des Atlas Sieger und Vučnik in ihren Besprechungen dem lebhaften Wunsch nach einer Veröffentlichung der Grenzbeschreibungen Ausdruck gegeben und Sieger hofft, dass ,deren Sammlung und kritischer Ausgabe durch die Studien zum Atlas wohl wesentlich vorgearbeitet" sei ${ }^{2}$ ).

Wie gestaltet sich nun diesen Wünschen und Plänen gegenüber die praktische Ausführung? Für Steiermark hatte Mell seiner Arbeit über den comitatus Liupoldi als Probe den Abdruck einer Reibe von einschlägigen Grenzbeschreibungen beigegeben. Darüber hinaus ist, da "Abhandlungen" über das steirische Gebiet bisher nicht zur Ausgabe kamen, im Rahmen der Atlasunternehmungen nichts weiter von dieser Art publiziert worden. Die Erläuterungen Pircheggers führen zwar Schritt für Schritt Datum und Fundort der benützten Beschreibung nebst Archivsignatur an, aber sie teilen nur selten kurze Stellen aus ihnen mit; dieser konsequent eingehaltene Vorgang berührt den Benützer aufs angenehmste, er trägt wesentlich dazu bei, eine übersichtlich kuappe Fassung der Erläuterungen zn ermöglichen, und er ist für Steiermark insofern sehr angemessen, als die überwiegende Mehrzahl der für dieses Land in Betracht kommenden Grenzbeschreibungen, im steirischen Landesarchiv vereint, ziemlich leicht zugänglich ist. Für Salzburg war von Richter schon im Jahre 1885 der Anfang zu einer Sammlung der Grenzbeschreibungen gemacht worden, indem er, das einschlägige Material der "Taidinge" ergänzend, im Anhang zu seinen Untersuchungen mehrere Texte abdruckte ${ }^{3}$ ); einige andere Stücke sind von ihm in den Erläuterungen mitgeteilt worden, mehrere "Rügungen", die anch über die Grenzen des Salzburger Stadtgerichtes Aufschluss gewähren, waren zum Abdruck in jenen Teilen der Abhandlungen bestimmt, deren Erscheinen Richters Tod verhindert hat. Bedeutende quantitative Fortschritte hat in den letzten Jahren nur die Publikation der Grenzbeschreibungen gemacht, die sich auf heute oberösterreichischen Boden beziehen; Strnadt erkannte sehr gut die Wichtigkeit dieser Quellen und war bestrebt, sie zugänglich zu machen, indem er vieles hieraus schon in den Erläuterungen mitteilte, die Hauptmasse aber in den Abhandlungen unterbrachte; ja das

1) Ebenda 6, 63 .

2) Mitt. der geogr. Gesellschaft 1907, S. 243 und Ztschr. des historischen Vereined f. Steiermark 4, 227.

3) Mitt. des Inst. 1. Ergbd., $719 \mathrm{ff}$. 
Streben, sie in den Text einschalten oder doch in Aumerkungen oder Beilagen mitteilen zu können, dürfte in gewisser Hinsicht für die Anlage seiner Abhandlungen massgebend geworden sein 1). Das verdient insofern den Dank der Benützer, als die Grenzbeschreibungen für Oberösterreich lange nicht so bequem zugänglich sind, wie etwa die für Steiermark; Strnadt hat viele von ihnen aus privaten Archiven aus licht gezogen. Leider ist es ihm aber nicht geglückt, die löbliche Absicht in einer praktischen, dem Bedürfnis entsprechenden Form zu lösen. Störend ist zunächst schon der verschwenderische Gebrauch grosser Aufangsbuchstaben, die doch gerade in so namenreichen Quellen unbedingt für die Namen zı reservieren wären ${ }^{2}$ ), sehr bedenklich der Gebrauch von Klammern sowohl für in den Text der Quellen eingeschobene Erklärungen des Herausgebers als auch für die zu dem Text gehörigen Parenthesen ${ }^{3}$ ), sehr uubequem auch, dass manchmal bei den als Beilagen der Abhandlungen gedruckten Stücken die Quellenangabe den Aufbewahrungsort nicht neunt, so dass man ihn erst an andern Stellen suchen muss't). Alle diese Dinge würden ohne Zweifel ein-

1) S. oben S. 578 Anm. 2.

2) Strnadt scheint bei einem Teil der abgedruckten Quellen alle Hauptworte gross geschrieben zu haben, (so zumeist wohl Archiv 99, 222 bis 296), teils folgt er dem regellosen Gebrauch seiner Vorlagen (so werden sich die Schwankungen bei den im Archiv 94, $614 \mathrm{ff}$. und 99, $297 \mathrm{ff}$., und natürlich auch die beim Abdruck einzelner deutscher Urkunden, z. B. Arch. 99, 205 ff. erklären); dabei geschieht es dass gelegentlich auch einzelne Eigennamen klein gedruckt werden (Erläut. S. 15, 20 f.).

3) Man vgl. otwa Arch. 99, 227, 247, 258, wo Klammern von beiderlei Bedeutung nebeneinanderstehen, dann ebenda $240 \mathrm{ff}$, $248 \mathrm{ff}$, $252 \mathrm{ff}$,, wo es sich meistens um Erklärungen des Editors handelt, 307, 309 f., wo wieder Textparenthesen so bezeichnet sind, und versuche dann im Arch. 94, 624 ff. von Fall zu Fall zu erraten, was die Klammer bedeutet. Von Arch. 94, 631 an sind dann zum Glück für Errklärungen eckige Klammern angewendet, aber die nützliche Neuerung (der doch völlige Ausschaltung aller Erklärungen aus dem Text und Einreihung in die Fussnoten weitaus vorzuziehen wäre) ist, wie obige Beispiele zeigen, im 99. Band wieder in Vergessenheit geraten.

4) Gleich das erste Stück im Anhang der zweiten Strnadt'schen Abhandlung, Arch. 94, 614, trägt den sparsamen Quellenvermerk, Libell in einer Abschrift XVII. sec."; wer nun etwa glauben würde, den Auf bewahrungsort in der am Schlusse derselben Nummer angebrachten Bemerkung angegeben zu finden, der käme in die Irre; die dort stehende Archivnotiz (Schlossarchiv Greinburg) bezieht sich nur auf die von Strnadt seiner Beilage I angehängte Urkunde von 1347; Beilage I aber liegt im Archiv Losensteinleiten Lade 62 Fasc. I Nr. 5, wie aus Erläut. S. 14 zu ersehen. Bei Beilage VI (Arch. 94, 624) ist wohl am Schluss die Folioangabe gesetzt, aber am Anfang fehlt der Fundort, der freilich zu erraten und Erläut. S. 14 auch genannt ist. Zu Beilage XVII (Arch. 94, $649 \mathrm{ff}$.), wo Strnadt wieder 
beitlicher und besser geordnet worden sein, wenn die Grenzbeschreibungen an einer einzigen bestimmten Stelle gedruckt und nicht an drei verschiedenen, teils in den Erläuterungen, teils im Text der Abhandlungen, teils als Anhang zu den letzteren, zersplittert worden wären. Solche Behandlung einer Quellengattung, die für das ganze grosse Unternehmen der Wiener Akademie ron grundlegender Bedeutung ist, muss entschieden als ein ernster Missgriff bezeichnet werden. Die Zersplitterung des zusammengehörigen Quellenmaterials ist in diesem Fall umso bedauerlicher, als ja die Grenzbeschreibungen ihrer Natur nach nicht blos für das eine Gericht, das sie zum Mittelpunkt nehmen, sondern fast immer auch für die Geschichte mehrerer Nachbargerichte in Betracht kommen; wenn man sie also nicht an besonderer Stelle vereinigt, sondern , in den Text verwebt", wie Strnadt es (Arch. 99, 4) nennt, so müsste eigentlich jede an mehreren Stellen des Textes, sei es der "Erläuterungen" oder der "Abhandlungen", angeführt und zu diesem Zweck in ihre Teile zerrissen werden 1 ). Will man dem entgehen, die Grenzbeschreibungen aber doch mit dem Text verbinden, so bleibt wohl nichts übrig, als sie in den Anmerkungen unterzubringen; das hat Strnadt in seinen beiden ersten Abhandlungen getan, dabei sind ihm aber die Anmerkungen infolge der oft sehr umfänglichen Quellenwiedergabe so sehr über den Text hinausgewachsen, dass alle Übersicht verloren geht. Der Text nimmt auf manchen Seiten nur ganz wenige Zeilen ein, während sich unter dem Strich endiose Fussnoten dahinwälzen, die dann selbst wieder neue Anmerkungen

die Archivsignaturen aber nicht den Fundort vermerkt, vgl. S. 585 Anm. 2 und 586 Anm. 3. In Anhang der dritten Abhdlg. (Arch. 99, $291 \mathrm{ff}$.) haben Nr. II, III, IV teils gar keine teils unzureichende Quellenangaben; man ergänze sie bei II und III aus Arcb. 99, $225 \mathrm{Anm} .1$ und Erläut. S. 19, wo übrigens zu ersehen, dass diese Stücke (II vollständig, III zur Hälfte) schon bei Pillwein, Beschreibung der Provinzialhauptstadt Linz (1824) S. $58 \mathrm{ff}$. gedruckt sind, was doch jedenfalls in der Überschrift des Neudruckes auch zu el:wähnen gewesen wäre; Beilage IV stammt aus dem oberösterr. Landesarchiv zu Linz, vgl. Arch. 99, 278 Anm. 1 und Erläut. 21.

1) Beispiele zerrissener Beschreibungen bieten sowohl Strnadts Erläut. (S. 20, 2. Sp. werden zwei Stellen aus dem Urbar von Kammer 1550/51 mitgeteilt; in die Lücke binein gehört, ohne sie ganz zu füllen, die ebenda S. 21, 2. Sp. mitgeteilte Stelle), wo stückweise Mitteilung der Beschreibungen, wenn schon überhaupt nötig, eher zu entschuldigen, als besonders die 3. Abhandlung Strnadt's. Arch. 99, 230 steht ein Stück der Peuerbacher Grenzbeschreibung von 1617, die dann S. $240 \mathrm{ft}$. mit Ausnahme dieser Stelle vollständig folgt; ebenso ergänzen sich die Starhembergische Beschreibung ron 1592/5 aus S. 247 f. u. $255 \mathrm{ff}$; die Tegernbacher von c. 1656 aus 248 und 252 ff.; die jüngere Starhembergische aus $\mathrm{S} .257 \mathrm{ff}$., $263 \mathrm{f}$. und $267 \mathrm{f}$. 
zur Welt bringen oder vorübergehend anderen kürzer gestalteten Schwestern Platz machen und erst sobald diese zu Ende sind, ihren Lauf fortsetzen und vollenden ${ }^{1}$ ). Dazu kommt, dass manche Stellen von Grenzbeschreibungen doppelt, nämlich in den Erläuterungen und in den Abbandlungen, gedruckt sind ${ }^{2}$ ) und dass, wo man mit Hilfe solcher Parallelstellen eine Kontrolle üben kann, sich mancherlei kleine Verschiedenheiten herausstellen, die das Vertrauen in die Zuverlässigkeit der Texte etwas vermindern ${ }^{3}$ ), endlich dass in den viel früher gedruckten Erläuterungen natürlich noch nicht auf die $A b$ bandlungen verwiesen werden lrounte, so dass es dem Benützer überlassen bleibt, zu suchen ob die in den Erläuterungen erwähnte Grenzbeschreibung vielleicht irgendwo in den Abhandlungen ganz oder teilweise, einheitlich oder stückweise zu fiuden wäre. Wer auf diese Art die Grenzen auch nur einiger Landgerichte an der Hand der Quellen zu studieren unternimmt, der überzeugt sich schnell und gründlich von diesen Übelständen.

Gewiss ist ja die grosse Mühe anzuerkennen, welche Strnadt auf das Sanmeln der Beschreibungen und auch darauf verwendet hat, sie in seine Ausführungen auf die eine oder andere Art einzuschachteln. Und auch daran ist zu erinuern, dass er diesen für ihn selbst recht schwierigen Pfad gerade zu dem Zweck eiuschlug „um dem örtlich ferner stehenden Forscher die Übersicht der ursprünglichen Markungen

1) Vgl. Archiv 94, 286 bis 290,297 bis 302, 596 bis 598, Archiv 99, 242 f.; ähnliche Erscheinungen trifft man aber aus anderen Anlässen auch sonst bei Strnadt, Arch. 99,47 bis 53, 74 bis 76, 88 bis 90,193 bis 195, 204 bis 209.

2) So Erläut. S. 11 und Arch. 94, 284 dieselbe Stelle der Mauthausener Urbare, Erläut. S. 19, 1. Sp. unten, 20, 2. Sp. unten, 21, 2. Sp. unten dieselben Stellen wie Arch. 99, 222, 272 und $280 \mathrm{f}$.

3) Wenn Erläut. 11 von Arch. 94, 284 abweicht (Lindenstain: Lindenstamm, derselben: demselben, Marichbach: Marchbach, volgendts wider: volgendts gestracks wider), so bleilt möglich, dass das einemal das Urbar von 1489, das anderemal dasjenige von 1558 benützt wäre. An den anderen Stellen muss jedes. mal dieselbe Vorlage benützt sein; abex es fehlen doch Erläut. S. 19, 1. Sp. unten nach ,purckfrid‘ die Worte ,der vest Mistlbach', S. 20, 2. Sp., Z. 17 v. unten nach ,stadlfürst' die Worte ,vom Pettenfürst'; statt ,Eisenpunkenguet', ,Krien', ,Kienperg', ,Galingpuechen' (Arch. 99, 272) beisst es Erläut. 20, 2. Sp. unten: Eisen Pauckhen guet, Khien, Khien Perg, Galling Puechen. In der Wildenecker Rügung, deren voller Wortlaut für die noch ausständige letzte Abhandlung aufgespart ist und von der man vorläufig aus den Erläut. S. 21, 2. Sp. unten das Jahr (1462), dagegen aus Arch. 99, 280 Anm. 4 die Archivsignatur und den Fundort erführt (es wird wohl hier und dort dieselbe Rügung gemeint sein), stehen sich die Lesarten Undrachswalt: Untrachswald, regenwasser: wasser und Rockens Peunt: Rogkenspeunt gegenüber. 
zu erleichtern"1). Aber ich denke, diese Übersicht würde durch vollständige Loslösung der Grenzbeschreibungen vom Text der Abhandlungen viel besser erreicht werden und dieser zweite Weg würde noch weitere Vorteile bieten, die auf dem ersten nicht zu erreichen sind. Es liegt ja in der Natur der Sache, dass Grenzbeschreibungen desselben Gerichts sich za einander häufig verhalten wie Vorurkunde und Nachurkunde oder auch wie verschiedene Überlieferungsformen derselben Quelle, dass sie also in vielem übereinstimmen und gerade die Abweichungen das besondere Interesse erregen. Warum sollten nicht also auch auf sie die bei anderen Quellen längst gebräuchlichen Mittel angewendet werden, um solches Verhältnis zu veranschaulichen: Zugrundelegung des besten Textes, wo sich die Varianten der anderen unter dem Strich übersichtlich unterbringen lassen ${ }^{2}$, oder Nebeneinanderstellen der beiden Fassungen in zwei oder mehreren Spalten, wo sich stellenweise Übereinstimmung, stellenweise aber grössere Abweichung ergibt ${ }^{3}$ ), endlich selbständiger Abdruck der beiden Formen und Kennzeichnung der Entlehıungen durch Petitdruck, wo veränderte Anordnung oder sonstige Umstände einen anderen Modus nicht zulassen ${ }^{4}$ ). Solange man die Grenzbeschreibungen „in den Text verwebt", muss man auf solche Veranschaulichung ihres gegenseitigen Verhältnisses verzichten, erst sobald man sie vom Text loslöst, als Beilage und Anhang zu den Abhandlungen oder als besondere Sammlung, lässt sich eine ihrem Wesen entsprechende Editionsart ausbilden. Und dabei würde endlich auch noch eine Hauptsache, die Erklärung der Ört-

1) Archiv 99, 4.

2) So wären z. B. die beiden Grenzbeschreibungen der Herrschaft Wildenstein, die jetzt Arch. 94, 631 in extenso hintereinander stehen, aber aufs engste übereinstimmen, zusammenzuziehen gewesen. Ein Muster bietet Mell in den Mitt. des Inst. 1, $438 \mathrm{Nr}$. 8. Vgl. auch Forst, Das Fürstentum Prüm (Erläuterungen zum geschichtlichen Atlas der Rheinprovinz 4. Bd.) S. $91 \mathrm{ft}$.

$\left.{ }^{3}\right)$ Diesen Weg hat schon Mell a. a. O. $440 \mathrm{ff}$. in einem Fall eingeschlagen; reichlichen Gebrauch vom Spaltendruck machten auch Handel-Mazzetti, Das Gemärke von Wildberg, im 57. Jahresbericht des Museums Franzisco-Carolinum in Linz (1899) S. 65 ff. und Fabricius, Das Hochgericht Rhaunen (Erl. z. gesch. Atl. der Kheinprovinz 3. Bd.) $34 \mathrm{ff}$; vgl. aber auch die ebenda S. $18 \mathrm{ff}$. angewandte Methocle.

4) Vergleicht man etwa die beiclen im Arch. 99, $292 \mathrm{ff}$. gedruckten Beschreibungen des Ldg. Donautal, so ergibt sich enge Übereinstimmung der angeführten Grenzmarken, aber die Reihenfolge in der sie genannt werden, ist verschieden, die Begehung erfolgte in entgegengesetzter Richtung. Eine kurze Andeutung der Richtung und des Anfangspunktes (z. B, , von S. über W.N.O, fortschreitend - od. dgl.) würde, jeder Grenzbeschreibung beigegeben, dem Benützer sehr willkommen sein. 
lichkeiten, welche jede Grenzbeschreibung anführt, zu ihrem Rechte kommen. In dem Aufsuchen der Siedlungen und Wege, Gewässer, Berge, Wälder, einzelner Bäume und lünstlich gesetzter Marksteine, welche die Grenzbeschreibungen nennen, liegt ja ein grosser Teil der wissenschaftlichen Leistung, welche die Atlasbearbeiter auf sich nehmen; von ihrer zutreffenden Bestimmung hängt die richtige Auffassung des Grenzverlaufs und der Gerichtsverhältnisse und die wahrheitsgetreue Zeichnung der Karte ab. Es ist daher unerlässlich, dass dem Benützer über diese Bestimmungsarbeit auch Rechenschaft gegeben werde, und das kann am besten in Form von fortlaufenden Fussnoten zu dem Abdruck der Grenzbeschreibungen geschehen ${ }^{1}$ ).

Es möchte vielleicht eingewendet werden, die Beigabe von solchen Ortserklärungen sei nicht so wichtig, weil ja die Karte selbst diese Erklärungen in sich schliesse und in klarster Form veranschauliche. Und in der Tat, das Kartenbild muss ganz wesentlich mithelfen, die Grenzbeschreibungen zu erläutern, und die Karte muss, so viel als möglich, auch dem Benützer die Kontrolle dafür an die Hand geben, dass die Grenzen richtig und genau auf Grund der Quellenangaben gezeichnet sind. Natürlich sollen die Ortlichkeiten, die als Grenzpunkte in der Quelle überliefert sind, soweit es der Raum zulässt, auch in der historischen Karte an richtiger Stelle verzeichnet werden. Finden sich nun diese Punkte schon in den dem Atlas zugrundegelegten neuen Kartenwerken, also auf der Generalkarte selbst (1:200.000) oder auf der Spezialkarte (1:75.000), welch letztere man wohl überall mit zu Rate ziehen muss, mit derselben oder doch einer nahe verwandten Namensform eingetragen wie in der Quelle, so ist die Kontrolle gegeben, der Beweis geschlossen ${ }^{2}$ ). Aber die Grenzbeschreibungen enthalten ein viel grösseres Namenmaterial und es musste daher eine grosse Zahl von Namen entlang der Landgerichtsgrenzen eingetragen werden, welche selbst auf der Spezialkarte fehlen ${ }^{3}$ ). Wer

1) Ein treftliches Vorbild bietet in dieser Hinsicht Forst in den Beilagen seines Buches, Das Fürstentum Prüm S. 81 bis 144, der jeden Namen der Grenzbeschreibungen, so gut es geht, erklärt und dabei in jedem Fall das verfügbare Kartenmaterial (Messtischblätter, Flurkarten und sonstige Detailkarten) anführt.

${ }^{2}$ ) Auf Bl. 9 vermisse ich den auf der Spezialkarte eingetragenen Namen „Gr. Plaike* (1032 M.), also denjenigen Höhenpunkt in dem rom Zifanken gegen Colomanstaferl streichenden Bergrücken, an welchem die Grenze der Ämter Henndorf und Köstendorf von der Neumarkt-Wartenfelser Ldg.-Grenze abzweigen sollte; die Plaike ist als Grenzpunkt der Henndorfer Rieget erwähnt in den Salzburgischen Taidingen S. 14 .

3) So z. B. in der westlichen Hältte des Blattes 9: Markstein im Brennerhölzl (im Schönramer Filz, zw. den Ldg. Oberlebenau, Raschenberg, Halmberg) 
nun die betreffende Gegend genan kennt, dem wird vielleicht diese Eintragung genügeu, weil ihm die Örtlichkeit und der Name geläufig sind; aber der Fernerstehende darf in allen Fällen irgend eine Aufklärung darüber erwarten, mit welchem Recht der in der Grenzbeschreibung vorkommende Name, den er auf der Spezialkarte nicht findet, gerade hier und nicht an etwas anderer Stelle eingetragen sei. Noch viel notwendiger aber wird solche Aufklärung an allen denjenigen Punkten, welche auf der historischen Karte nicht unterzubringen sind.

Die Bearbeiter des Atlas sind solchen Wünschen vielfach durch in den Text der Quellen eingeschaltete Erklärungen ${ }^{1}$ ) und auch durch Fussnoten oder Bemerkungen im Text der Erlänterungen oder Abhandlungen entgegengekommen, aber es ist immer noch keine leichte Sache den auf den Kartenblättern eingetragenen Grenzverlauf mit den Grenzbeschreibungen zu vergleichen. Einige Versuche, die ich in dieser Richtung vornahm, haben mich überzeugt, wie wünschenswert eine noch weitergehende Kommentierung der Grenzbeschreibungen wäre. So vermag ich auf Blatt 9 des Atlas, obwohl mit der Umgebung ron Salzburg sehr gut vertraut, dennoch den Verlauf der Grenzen des Stadtgerichts Salzburg nicht ganz zu verstehen, da die zugrundeliegenden Grenzbeschreibungen ${ }^{2}$ ) der Ortserklärungen entbehren. Jedenfalls verlaufen die beiden Partien der Grenze, die man auf dem Grundriss bei Hübner sieht ${ }^{3}$ ), etwas weiter nördlich, als sie Richter gezeichnet hat. Wenn es ihm vergönnt gewesen wäre, die Publikation der einschlägigen Rügungen ${ }^{4}$ ) zu vollenden, würde er diesen Sachverhalt gewiss aufgeklärt haben. Aber auch in solchen Teilen Oberösterreichs, wo Strnadts Arbeit in Karten, Erläuterungen und

Jungfernbr., Zellach-Alpe, Gurr-Wd., Weiss-Wd., Taderer-Schnee, Abfalter, Kühstein (sämtlich am Untersberg, wo auch Geiereck und Berchtesgadener Hochthron mit anderen Namen bezeichnet werden mussten), Reissende Fahrt (bei Schwarzbachwacht), Kesselbrunn (am Gaisberg bei Salzburg), Rossbacbgut (beim Zusammenstosse der vier Gerichte Neuhaus, Wartenfels, Golling und Glaneck), Windisch Thörl (zw. Fuschl u. S. Gilgen), Grünausattel (zw. Wartenfels und Golling). Ähnliche und vielleicht noch zahlreichere Belege bietet jedes Blatt. Dagegen bedauert man, die in einer Gasteiner Grenzrügung, welche in den Erläut. S. 2 mitgeteilt ist, genannten Örtlichkeiten: Hechenwarter Alben, Herndl, Stinkoten, auf Blatt 17 nicht eingetragen zu finden; mit der Bemerkung »sämtliche Namen bekannte ist dem Leser nicht viel gedient.

1) S. oben S. 589 Anm. 3.

2) Die salzburgischen Taidinge S. 113 und Mitt. der Gesellsch. für Salzb. Landeskunde $28,418 \mathrm{ff}$.

3) Hübner, Beschreibung der Residenzstadt Salzburg 1 (1794).

4) S. Erläut. S. 4, 1. Sp. unten. 
Abhandlungen schon abgeschlossen vorliegt, erheben sich mir Zweifel, die ich, obwohl hier nicht so genau mit den Örtlichkeiten vertraut, doch nicht übergehen möchte. Auf Blatt 5 zieht Strnadt die Grenze zwischen dem Landgericht Schwertberg, oder genauer gesagt, zwischen dem im Jahre 1644 an Schwertberg abgetretenen Teil des Ldg. Mautbausen auf der einen und dem Ldg. Haus auf der auderen Seite von Obenberg in südwestlicher Richtung über die Höhen zur Donau. Nach den Grenzbeschreibungen des 15. und 16. Jahrhunderts ${ }^{1}$ ) sollte man annehmen, dass sie im Tal den Marbach entlang gegangen wäre, wie auch Strnadt (Erläut. S. 11, 1. Sp.) erklärt; an anderer Stelle ${ }^{2}$ ) sagt er allerdings dass ,späterhin wenigstens" der Marbach ,nicht mehr durchwegs die Grenze“ bildete; a.ber indem er dafür, soviel ich sehe, keinen Quellenbeleg beibringt, erscheint dem Benützer die von Strnadt eingetragene Grenze unsicher. Auch sehe ich nicht, ob mit Absicht oder nur aus Versehen die erwähnte Ortschaft Obenberg (BI. 5 ebda.) anders, und zwar um etwa $1 \frac{11 / 3}{\mathrm{~km}}$ weiter nordöstlich eingetragen wurde, als die Spezialkarte sie ausweist; nach dieser liegt Obenberg nicht in dem bei Ponneggen ausmündenden Tal, sondern auf der westlichen Höhe, an der Strasse von Mauthausen nach Pregarten. Dass ebenda die der unteren Aist folgende alte Grenze zwischen Riedmark und Machland unbezeichnet geblieben ist, hat mit Recht schon Sieger als Versehen erklärt3). Einer Erklärung bedürfte aber vielleicht auch der südliche Endpunkt dieser Grenze: von den Grenzbeschreibungen lässt sie die eine „in der Thonau zu Sebara bey der prugken" beginnen und die Aist hinaufgehen (Arch. 94, 284), die andere (Erläut. S. 9, 1. Sp.) nennt Ober-Seebern als den Punkt, wo die Aist in die Donau rinnt. Strnadt spricht an der letztgenannten Stelle wohl mit Recht von Untersebern und trägt dieses in die Karte ein, an der anderen aber von Obersebing, womit wol das jetzt mehr als $1 \mathrm{~km}$ von der Aistmündung entfernte Obersebern gemeint wäre. Eine Differenz in der Einzeichnung der Ortschaften ergibt sich ferner Bl. 4 rechts unten, an der Nordspitze des Ldg. Puchheim, wo nach der Spezialkarte Penetsdorf nicht an dem gegen Schwanenstadt ausmündenden, im Oberlauf durch den roten Überdruck ${ }^{4}$ ) zugedeckten

1) Archiv 94, 284 nach Urbaren von 1489 und 1558, ebenda 286 Anm. 2 nach einem 1804 kopierten Urbar von 1590.

2) Archiv 94, 284.

3) Mitt. der geogr. Gesellsch. 1907, 255 Anm. 2.

4) Über die Vorteile und Nachteile des erst nach Richters Tod in die Karten eingeführten roten Überdrucks vgl. Sieger a. a. $0.252 \mathrm{ff}$; es ist natürlich, aber doch sehr zu bedauern, dass gerade auf den salzburgischen Blättern (9 und 17), 
Bach, sondern im selben Tal wie Wufing, etwa 600 M. nordnordwestlich von diesem Ort gelegen ist. Verschiebt sich dadurch der in der Wartenburger Grenzbeschreibung von 1718/20 (Archiv 99, 303) dargelegte Grenzzug zwischen Wartenburg und Puchheim schon etwas nach Osten, so scheint mir mit dem weiteren Wortlaut derselben Stelle auch die Fortsetzung dieser Grenze auf Blatt 4 nicht übereinzustimmen; da die Puchheim-Wartenburger Grenze „hinab auf UnterTalhamb, verners durch dises dorf der strassen nach .. sodann in diser strassen fort auf Nider-Pengering und Nider-Holzhamb" ging, so kann kaum bezweifelt werden, dass sie von Penetsdorf (nördlich Wufing) gegen Osten lief, ungefähr dort, wo Strnadt die PuchheimStarhembergische Grenze zeichnet, dass sie dann ron N.-Talheim aus bis N.-Holzham genau dem Zug der noch jetzt bestehenden Schwanenstädter Strasse folgte; dadurch erhält man hier denselben Verlauf, den nach der Einzeichnung in Blatt 4 die bayrisch-österr. Grenze vom Jahre 1810 hatte. Was dort als nördlicher, gegen NW. vorspringender Zipfel des Puchheimer Gerichts erscheint, wird also 1718/20 ziemlich vollständig zu Wartenburg gerechnet. Da nun aber nach dem Starhemberger Urbar von 1660 (Archiv 99, 268) das Puchheimer Ldg. dennoch bis zum "Rambkhor" hinaufgereicht hat, so muss wohl auch die Puchheim-Starhembergische Grenze, die vom Rankar über Iming

denen in diesem Zeitpunkt Richters sachkundige Hand fehlte, verhältnismässig viel Fehler mit diesen Farbenlinien begangen wurden; zu den von Sieger angeführten Beispielen kommt nämlich noch das Hehlen einer roten Linie, die am linken Rand von Bl. 9 westlich vom Gipfel des hohen Staufen dem zur Zwieselspitze hinüberführenden Grate folgen sollte; es fehlt hier allerdings auch die schwarz vorgezeichnete Ldg.-Grenze wohl deshalb, weil es sich um zwei bairische Gerichte handelt; Strnadt aber hat auf Bl. 4 und $1^{2}$ die roten und die schwarzen Gerichtsgrenzen noch weit nach Baiern hineingeführt. - Das oben erwähnte Beispiel gibt indes Anlass, auf einen von Sieger nicht berührten und doch recht wesentlichen Ǹachteil der roten Linien hinzuweisen: sie verdecken leider häufig die Wasserläufe, denen die Grenze folgt. In vielen Fällen haben die Zeichner dieses Übel zu vermeiden gestrebt, indem sie blaue, schwarze und rote linien nebeneinander setzen, wie das z. B. auf Bl.5 an der Mühl, Rodl, Aist und Naarn und auch aut dem steirischen Gebiet meist sehr sorgfältig durchgeführt ist. Aber allzu weit sollten die Grenzen nicht auseinandergezogen werden, wie dies etwa auf Blatt 18 rechts unten geschehen ist, wo die nur mit Unterbrechungen eingetragene Signatur der Landesgrenze Anlass gegeben zu haben scheint, die rote Linie ziemlich willkürlich ron dem Rossbach (Gr. Pretalbach) zu entfernen. Für vollständiges oder teilweises Verdecken der Wasserläufe durch die rote Grenze bieten Bl. 4 (Gurtenbach, Ampflwangerbach), Bl. 9 (die Vöckla bei Frankenmarkt, Schwarzbach von Ebenau abwärts, Weisbach am Untersberg) Beispiele. Die Sache wird besonders dann störend, wenn der Bach- odex Flussname nicht beigeschrieben oder doch nur an einer Stelle eines längeren Laufes zu finden ist. 
nach Herrenschützing läuft, etwas weiter nördlich gezogen werden, als sie Strnadt eintrug; wahrscheinlich deckte sie sich hier mit der Grenze des heutigen GB. Schwanenstadt, welche auf der Spezialkarte ersichtlich ist.

Zeigen diese Beispiele, dass eine bis ins einzelne gehende Ortserklärung nötig ist, um den Benützer vor Missverständnis und Misstrauen und vielleicht auch denjenigen, der die Grenzen einträgt, vor einzelnen Versehen zu berwahren, so sprechen doch selbst abgesehen von der Zuverlässigkeit der Grenzeintragungen andere Gründe dafür, die Mühe solcher Detailbestimmungen nicht zu sparen. Es stehen Namen von Naturobjekten in den Grenzbeschreibungen, die vor Jahrhunderten unmittelbar aus dem Mund des Volkes geschöpft, in den Karten und statistischen Werken nirgends gebucht, vom sprachlichen und volkskundlichen Standpunkt Aufbewahrung und Erklärung verdienen. Wer anders soll sie örtlich fixieren, wenn nicht der Bearbeiter des Atlas, der schon um der richtigen Erfassung der Grenzen willen, sie untersuchen muss? Die Anmerkungen zu dem Abdruck der Grenzbeschreibungen, denen schliesslich ein Namenregister hätte beigefügt werden können, würden dazu gute Gelegenheit geboten haben. Ein allzu starkes Anschwellen der Bemerkungen wäre etwa in der Weise zu vermeiden gewesen, dass in bestimmten, vorher bezeichneten Fälleu, also etwa wenn der Name zwar nicht auf der Spezialkarte aber in deu Katasterkarten oder anderen nambaft zu machenden Kartenwerken zu finden ist, đie Fussnote unterblieben wäre. Eine weitere Vereinfachung hätte allenfalls auch dadurch erreicht werden können, dass die Lage bestimmter Ortlichkeiten, für deren Namen die Karte nicht mehr Raum bietet, dort durch Einsetzung ron in bestimmter Reibe im Umkreis des betreffenden Landgerichts zu stellenden Ziftern zum Ausdruck käme. Aber gegen die Anwendung dieses Auskunftsmittels, welches von Fabricius bei seinen rheinischen Karten gelegentlich angewendet wurde ${ }^{1}$ ), spricht doch die Gefahr einer Überlastung der Karte. Es ist immer noch besser, wenn der Text der Grenzbeschreibungen von zahlreichen Anmerkungen begleitet wird, als wenn auf der Karte der ohnehin eng bemessene Raum an den Grenzen stellenweise durch Ziffern bedeckt und verdeckt würde.

1) So in cler Karte „Das Hochgericht auf der Heide in der Westdeutschen Zeitschr. 24, wo ein durchlaufendes Ziffernsystem von 1 bis 265 eingetragen und durch eine Legende im Text der zugehörigen Abhandlung erklärt ist. Die rot gedruckten Ziffern bedeuten hier teils Grenzpunkte, teils aber auch Grundflächen. Sie drängen sich namentlich im letztgenannten Fall sehr enge zusammen, verdecken das ohnehin allzu matt gehaltene Terrain und sind anch nicht immer 
Gerade an den Grenzen drängt sich ja vieles zusammen. Der Anschluss an die natürliche Bodengestalt soll hier mit noöglichster Deutlichkeit sichtbar sein, naan soll genau erkennen, wo und in welcher Art Grenzen zusammentreffen und von einander abzweigen. An manchen Grenzen gibt es dann überdies Streitigkeiten und Schwankungen, die sich durch Jahrhunderte hinziehen, und es ist eine wichtige Sache, die Linien der verschiedenen Ansprüche und Vergleiche gut ersichtlich zu machen. Freilich handelt es sich dabei oftmals um Territorien von unbedeutender Ausdehnung und von überaus geringer wirtschaftlicher Bedeutung; wie etwa bei den zwischen Salzburg und seinen Nachbarn strittigen Gebirgsstreifen am Untersberg, Lattengebirge und Steinernen Meer, dann am Schober, Schafberg und bei Weissenbach am Attersee. Aber diese Kleinigkeiten sind für den Historiker doch von grossem Interesse. Gleichsam wie die letzten, kleiner und kleiner werdenden Wellenschläge, die einer grossen Flut folgen, zeigen sie die Nachwirkung grosser geschichtlicher Ereignisse an und bereiten vor auf das Kommen neuer Epochen. Die Vorschiebung der salzburgischen Grenze im Nordosten ist vielleicht eine indirekte Nachwirkung des bairischpfälzischen Erbfolgekrieges und der Finanznöte Maximilians ${ }^{1}$ ), die Grenzstreitigkeiten Salzburgs gegen Berchtesgaden ziehen sich vom 12 . bis in die Mitte des 19. Jahrhunderts²), sie verknüpfen sich mit bedeutenden Vorgängen und deuten zugleich die Kontinuität von Kräften an, welche unter der Hülle von Friedensschlüssen und Verträgen fortwirkend unsere Zeit mit der des frühen Mittelalters verbinden. Und die geschichtliche Bedeutung der Grenzstreitigkeiten geht mit einem starken methodischen Interesse Hand in Hand. Gerade bei strittigen Grenzen beginnen die Akten am ehesten beredt und die Karten schon frühzeitig akkurat $/ \mathfrak{u}$ werden, so dass man bei Bestimmung der Örtlichkeiten und Ergründung der für den Wechsel massgebenden Ursachen hier auf besseren Erfolg zu rechnen hat als anderswo. Aus diesen Gründen würde es sich gewiss verlohnen, solche kritische Stellen, die auf der Landgerichtskarte nicht deutlich genug darstellbar $\operatorname{sind}^{3}$ ), auf

leicht aufznfinden. Sehr praktischen Gebrauch von den roten Orientierungs-Ziffern macht Fabricius auf zwei Karten des rheinischen Atlaswerkes, bei denen es gilt zweierlei politische Verhältnisse, Kreiseinteilung und territoriale Zugehörigkeit, zugleich zu veranschaulichen, nämlich auf den Blättern „Die Rheinprovinz i. J. 1789, Übersicht der Kreiseinteilung * und auf dem Karton zu Bl. V, wo die Territorien auf dem Hunsrück und im Nahegan dargestellt sind.

1) S. oben S. 568 Anm. 1.

2) Richter in den Mitt. des Inst. 1. Ergbd. 666 ff. und Erläut. S. 6.

3) Über Versehen, denen gerade die mit doppeiten oder dreifachen Grenzlinien dargestellten Gebiete besonders leicht ausgesetzt sind, wenn der Masstab. 
besonderen Nebenkarten (Kartons) in grösserem Masstab wiederzugeben, ein Vorgang der ja bei dem rheinischen Atlas in so vielen Fällen eingehalten worden ist ${ }^{1}$ ) und doch auch dem Unternehmen der Wiener Akademie gewiss nicht unerschwingliche Kosten auferlegen würde. Mit Nebenkarten kleineren Masstabs, wie sie ebenfalls auch im rheinischen Atlas mit gatem Erfolg angewendet worden sind2), hat schon Strnadt, indem er die Ausgestaltung des Landes o. d. Enns auf Blatt $1^{\text {b }}$ in dem Masstab 1:1,000.000 zusammenfasste, einen Anfang gemacht und mau darf wohl erwarten, dass auch die anderen Kronländer in ähnlicher Weise bedacht werden sollen; für Steiermark und Görz-Gradiska sind solche Pläne schon in dem Vortrage Mells im Herbst 1904 angekündigt worden ${ }^{3}$ ), für Salzburg werden sie durch

für die Differenzen unzureichend ist, vgl. Siegers Anmerkungen a. a. 0. $252 \mathrm{f}$. Fraglich ist mir auch ob die rote Linie am Schober (B]. 9), zwischen Ldg. Wartenfels und Wildeneck, richtig gezogen ist; sie folgt teils einer Ldg.-Grenze, teils der jetzigen Landesgrenze; Richters Erläut. S. 4 erwähnt hier einen Grenzstreit, über den 1751 bis 1758 verhandelt wurde, ohne dass seine Worte die Sache aufklären würden. Die Unmöglichkeit genauer Darstellung wird übrigens von Ricbter (ebenda) auch bei den verwickelten Grenzverhältnissen in der Gegend von Ebenau und Plainfeld hervorgehoben. Das von Sieger a. a. O. 247 vorgeschlagene Auskunftsmittel, ein Reinexemplar der Arbeitskarte, als welche die Blätter der Spezialkarte I : 75.000 dienen, an bestimmtem Ort zugänglich zu machen, wird doch naturgemäss nur einem engen Kreis von Benützern zu Gute kommen.

1) Der Karte der politischen und administrativen Einteilung der Rheinprovinz 1789 (7 Blätter 1:160.000) gab Fabricius acht Kartons in grösserem Massstab (fünf $1: 40.000$, je eine $1: 20.000,70.000$ und 80.000) bei; die von deniselben Autor bearbeitete Karte der kirchlichen Organisation und Verteilung der Konfessionen 1610 (4 Blätter $1: 250.000$ ) enthält auch Pläne von Trier und Köln, die in $1: 12.000$, bezw. ungefähr $1: 18.000$ gezeichnet sind. Auch Karg-Bebenburg sagt in den Forschungen zur Gesch. Bayerns 13, 264, indem er ein Herabgehen auf einen kleineren Masstab als $1: 200.000$ als für das geplante buirische Unter. nehmen untunlich erklärt, , man wird ohnedies wahrscheinlich nicht umhinkönnen, besonders verwickelte Verhältnisse auf Nebenkärtchen sehr grossen Masstabs zu verweisen ${ }^{\star}$.

2) Fine Übersicht gibt Beschorner in der Hist. Vierteljahrschr. 9, $20 \mathrm{f}$. Die drei hierhergebörigen Nebenkarten zur kirchl. Organisationskarte sind 1:1.000.000 (nicht $1: 100.000$ ) gezeichnet.

3) S. Deutsche Geschichtsblätter 6,57 und 61; war damals der freibleibende Raum auf den Blättern 30 und 33 (der älteren Zählung) für diese Nebenkarten reserviert, so ist jetzt, wohl infolge der Einschiebung von $\mathbf{1}^{\mathrm{a}}$, die Zählung geändert und der früher für die Entwicklung von Steiermark in Aussicht genommene Platz nicht mehr verfügbar. Man wird eben dann durch Einschiebung neuer Blätter Raum schaffen müssen, wie ja auch Richters Bemerkung Erläut. S. II andeutet. Vgl. auch Sieger a. a. 0. 260. - Die sieben im Masstab $1: 1.000 .000$ auf eine Karte mit modernem Weg- und Eisenbahnnetz und modernen Ortssigna. turen eingetragenen Darstellungen der territorialen Entwicklung von Oberöster- 
dessen selbständige, reichsfürstliche Stellung dringend erfordert'1) und man möchte wünschen diese. Blätter möglichst im Anschluss an die betreffenden Teile der gesamten Landgerichtskarte zur Verfügung zu haben. Für den wissenschaftlichen Wert des Atlas sind aber Detailkarten in grossem Masstab noch weit wichtiger, als diese zu erhoffenden Übersichten. Indes auch sie werden niemals in so ausreichendem Mass geboten werden können, dass dadurch die Kommentierung der abzudruckenden Grenzbeschreibungen wesentlich entlastet würde.

V.

Den Verlauf der Greazen von Landgerichten festzustellen, das ist ja schliesslich auch nicht das einzige, was den Bearbeitern der Landgerichtskarte obliegt. A ndere kartographische A u fgab en hängeu damit so enge zusammen, dass sie nicht auf ein späteres Stadium des Atlasunteruehmens hinausgeschoben werden, soudern womöglich zugleich mit dem Text und den Zeichnungen der Landgerichtskarte erledigt werden sollten. Neben den Grenzen der hohen Gerichtsbarkeit sind in reichem Masse und mit besonderer Signatur auch die Grenzen von solchen Gerichtsbezirken eingetragen worden, denen die Blutgericbtsbarkeit fehlte, also Hofmarken, Burgfriede oder ähnliche Bil-

reich und Salzburg, welche Strnadt in seinem 1907 ausgegebenen ,Historischen Schulatlas für Oberösterreich und Salzburg" (Wien, Hölzel, gebunden $3 \mathrm{~K}$ ) mit Erlänterungen herausgab, sind für Schulzwecke nützlich, befriedigen aber das wissenschaftliche Bedürfnis nicht.

1) Es muss, da die Überschriften der betreffenden Absätze in den Erläut. keinen Hinweis bieten, daran erinnert werden, dass das westlich an die untere Hölfte des Blattes 4 anstossende Rechteck (Zone 13 Col. VII der Spezialkarte) in den Rahmen der Atlasaufgabe fällt, weil es bedeutende Teile der Ldg. Tittmoning, Wildshut und Braunau und endlich auch den Burgfried der salzburgischen Stadt Mübldorf in sich schliesst. Anf das bairische Unternehmen zu warten oder ihm diese Teile überlassen zu wollen, wäre in diesem Fall wohl nicht angebracht, da es sich ja doch nur um eine kleine, von Richter zum grössten Teil schon geleistete Arbeit handelt; der Verzicht würde in diesem Fall auf das bairische Unternehmen gewiss nicht im Sinne cines Ansporns zu rascherer Erledigung wirken, wie das ja anderswo (vgl. Karg-Bebenburg in Forsch. z. bair. Geseh. 14, 258) zutreffen icag. Strnadt hat sowohl auf der Karte (Bl. 1a u. 4), als auch in den Erläut. S. 25 bis 27 und zum Teil auch im Archiv 94 die anstossenden bairischen Gebiete (wenn man von dem stiefmüttexlich bedachten Neuburg absieht, dem grosse Wichtigkeit zukommt) recht ausfïhrlich behandelt und dadurch denjenigen Forschern, welche um niederbairische und passauische Fragen bemüht sind, einen grossen Dienst geleistet. Es geht kaum an, diese f'örderung dem salzburgischen Geschichtsbetrieb vorzuenthalten, von dem doch schliesslich, da Richter an Zillner und Kleimayrn anknüpfte, der erste Anstoss zu dem ganzen Cnternehmen ausgegangen ist. 
dungen. Thre Eintragung war nötig, weil erst sie das Bild von der in einigen Ländern eingetretenen Zersplitterung der Justizverwaltung voll machen. Allerdings erwachsen aus diesem Teil der Aufgabe auch eigentümliche Schwierigkeiten, indem manchmal das Vorhandensein eines Burgfrieds bezeugt, seine Abgrenzung aber nicht festzustellen oder ihres geringen Umfanges wegen nicht darstellbar ist. Dabei ereignen sich auch Fälle, wo man schwanken kann, ob dem Bezirk die volle hohe Gerichtsbarkeit zustand, ob er also mit Landgerichtsgrenze eiuzuschliessen sei oder nicht ${ }^{1}$ ). Auch würde die Feststellung der Grenzen hier vielleicht eine Vertiefung in Archive erfordert haben, die zur Zeit vicht zugänglich waren. Im Territorium Salzburg, wo die Erzbischöfe mit Gewährung von Hofmarkgerichtsbarkeit sehr sparsam vorgingen, ergibt sich dagegen mehrfach die Gelegenheit, Unterabteilungen der Landgerichte auf der Karte einzutragen ${ }^{2}$ ). In engstem Zusammenhang mit der Darstellung der Gerichtsbarkeit steht natïrlich die Eintragung der Gerichtsitze und Schrannen, Hochgerichte (Richtplätze) und Burgen, für welche durchwegs besondere Signaturen, zum Teil sogar solche von verschiedeu abgestufter Bedeutung vorgesehen sind. Es wird niemand wundernehmen, wenn in dieser Hinsicht der lokalen Forschung noch mancher Nachtrag zu dem gelingen sollte,

1) Vou den Städten und Märkten in Oberösterreich sind Freistadt, Vöckla. bruck, Wels, Linz, Enns und Steyr mit Ldg.-Grenzen und rotem Überdruck umschlossen (bei Steyr fehlt allerdings an der ganz kurzen Westgrenze die rote Linie, sowie umgekehrt im Osten der Stadt ein kleines Stück des Ennslaufs irrigerweise von einer roten Linie begleitet ist). In Steiermark ist so nur Knittelfeld, in Salzburg sind nur Salzburg und (das ehem. salzb.) Laufen so behandelt; dagegen haben hier Hallein, dort Judenburg, Leoben, Bruck, Graz, Pettnu und Radkersburg Burgfriedgrenzen erhalten, obwohl doch mehrere von ihnen auch mit der Blutgerichtsbarkeit (Stock und Galgen) versehen waren. Bei Murau bemerkt Pirchegger Erläut. S. 34, dass sich der Burgfried nur innerhalb der Stadtmauern erstreckte; er ist also deshalb nicht eingetragen. Ob es bei dem salzburgischen Radstadt ebenso liegt, ist mir zweifelhaft. Auch der Burgfried des Markts Strasswalchen muss nach Hübner, Beschreibung des Erzstifts Salzburg 1, 207 gross genug gewesen sein, um eine Eintragung auf die Karte zu gestatten.

2) So in den Ldg. Haunsberg und Neumarkt (Bl. 9), Werfen (Bl. 9 und 17), Lichtenberg, Taxenbach (Bl. 17), Tamsweg (Bl. 17, 18); über die hierin bei Hauns. berg und Werfen begangenen Versehen vgl. Sieger a. a. O. 246 Anm. 1 u. 253 Anm. 1. Es wäre nahe gelegen hier, wo durch die Aufzählung in Kleimayrns Juvavia auch die einzelnen Rügete gegeben und bequem zu übersehen sind, diese in die Karte aufzunehmen; das war vielleicht Richters Absicht, indem er einen grossen Teil der betreffenden Narnen in clen Ldg. Neubaus und Wartenfels teils mit Ortsignatur, teils auch als Gegendnamen in die Karte eintrug bezw. sie aus seiner Vorlage, der Generalkarte, beibehalten liess. Aber die Absicht ist, wenn sie bestand, nicht allgemein durchgeführt worden. 
was der Atlas bietet; aber die Forderung, dass der Altlasarbeiter bei jedem Gericht diese für die Gerichtsentwicklung wichtigen Örtlichkeiten zu bestimmen trachte, sollte nicht aufgegeben werden, denn ihre Lage kann von entscheidender Bedeutung für die Erklärung der Gerichtsgeschichte werden ${ }^{1}$ ). Wenn alle diese Dinge konsequent in der Karte gebucht werden, so wird es, von wenig besiedelten Gebirgsgegenden abgesehen, kaum mehr allzu viel leere Stellen geben, die man durch Eintragung willkürlich gewählter Siedlungen aus Schönheitsgründen auszufüllen nötig hätte ${ }^{2}$ ). Dazu konmt, dass gewisse historischkartographische Forderungen, von denen die Atlaskommission bisher mit Bewusstsein abgesehen hat, weil sie eigene Vorarbeiten und Forschungen erfordert haben würden, vielleicht doch nicht auf die

1) Dass bei Goldegg (Bl. 17) anstatt der Signatur für den Sitz des Ldg. und der Hofmark, bei Bruckdorf (ebenda rechts unten) anstatt der Schrannensignatur und bei den seit 1356 bezw. 1680 nicht wieder hergestellten Burgen Liechtenthann und Altenthann (Bl. 9) Herrschaftssignatur angewandt ist, wird vielleicht auf Eintragungen zurückgehen, die vor der definitiven Festsetzung des Signaturensystems ertolgt waren. Blatt 5 enthält sowohl auf dem oberösterr. als auf dem niederösterr. Gebiet die Signatur des Galgens ziemlich häufig, wobei mir aber die Beisetzung des Wortes, Hochgericht * oder der Buchstaben ,H. G. * (bei Steyr) überflüssig zu sein scheint. Auf Bl. 9 fehlt der Galgen des Stadtgerichts Salzburg nördlich vom Kapuzinerberg, der auf der Stadtansicht von 1553 (reproduziert auf der Tafel zum 1. Bd. von Zillners Geschichte der Stadt Salzburg und auch schon bei Hübner, Beschreibung der Residenzstadt Salzburg 2. Bd.) rechts unten deutlich zu seben und genau bestimmbar ist, dann das Neuhauser Hochgericht bei Gnigl, dessen Lage Hübner (ebenda 1, 564) bezeichnet. Ferner ist die interessante Mitteilung des Henndorfer Schulleiters Franz Mayböck in den Mitt. der Gesellsch. f. Salzb. Landeskunde 33, 200 unberücksichtigt, welche die Lage des dortigen Galgens festzustellen ermöglicht; ebenso vermisst man in den Erläut. S. 2 einen Hinweis auf Pirkmayrs Notiz über Tanzlanbe und Pranger in Zell a. See (Mitt. der Gesellsch. 39, 28), die für die Frage, ob Zell oder Kaprun Gerichtssitz war, nicht ohne Wert ist; die, Tanzlaubes wird wohl nichts anderes als die alte Schranne gewesen sein.

2) Vgl. Sieger a. a. O. 263, der diesem ästhetischen Prinzip auch nicht zustimmt. Am Nordrand von Blatt 5 ist überflüssiger Weise auch eine Eisenbahnstation auf die Karte geraten, nämlich die von der Stadt weitabgelegene Station Freistadt („Bahnhof F.`); die Sache wird sich dureh ein Versehen bei der von Richter in den Deutschen Geschichtsblättern 4, 149 anschaulich beschriebenen Arbeit der Namenauswahl erklären, die jedenfalls sehr grosse Sorgfalt erfordert. Unklar bleibt mir ob es nicht möglich gewesen wäre, die Ämter des exempten Vizedomgerichtes zu Linz, über die Strnadt im Arch. 94, 609, 99, 17 und in Erläut. 18 handelt, auch mit einer besonderen oder der Schrannensignatur zu bezeichnen; bei einigen von ihnen ist Ortssignatur angewendet, andere sind auf der Karte gar nicht gebucht. Auch bei Ramingstein (Bl. 18) möchte man den Sitz des Berggerichtes gerne durcb eine Signatur angedeutet sehen. 
Dauer von der Landgerichtskarte ganz umgangen werden können. Ich meine die Eintragung von Strassen und Wegen, sowie die Berücksichtigung von Veränderungen im Lauf der Gewässer. Bisher sind die Flüsse und Bäche durch Beibehaltung des blauen Steins aus der Generalkarte herübergenommen, also nur nach dem gegenwärtigen Stand aus der Karte ersichtlich; das ganze Strassennetz hingegen ist weggelassen $\mathbf{1}$ ). Wie es scheint, wird in dieser Hinsicht der österreichische Atlas von verwandten Unternehmungen, die in anderen deutschen Landen in Gang kommen, doch nicht so sehr als Muster betrachtet werden, zum Teil wohl deshalb weil etwa in den östlichen preussischen Staaten diese Fragen wichtiger oder leichter zu lösen sind als in den Alpenländern. Aber auch hier kann man bei gewissenhafter Feststellung der Gerichtsgrenzen diesen Problemen eigentlich nicht ganz aus dem Wege gehen. Man hätte sie, so scheint mir, nicht in ihrer vollen Grösse in den Arheitsplan der Landgerichtskarte einbeziehen müssen, aber man hätte an den Punkten, wo Strassen, Wege und Wasserläufe als Gerichtsgrenzen dienen, nicht vor der Feststellung des alten Bestandes zurückscheuen sollen. Dort wo solches Zusammentreffen stattfindet, wird ja auch derjenige, dem es allein um das Weg-oder Flussnetz zu tun ist, gerade dieselben Quellen zurate ziehen müssen wie der Erforscher der Gerichtseinteilung; er wird enttäuscht sein zu sehen, dass dieser das Problem nicht gelöst habe, oder, was schlimmer wäre, er wird auf Grund der Eintragung in die Landgerichtskarte Fragen für gelöst ansehen, die tatsächlich noch nicht gelöst sind, weil man den erwähnten Schwierigkeiten auswich. Bei der im Zug befindlichen Bearbeitung der niederösterreichischen Landgerichte wird die Feststellung der alten, in den Grenzbeschreibungen wirklich gemeinten Flussläufe wohl ein schweres Stück Arbeit, aber keine undankbare Aufgabe bedeuten. Nicht blos der Geograph, auch der Historiker, der besonders bei Betrachfung kriegerischer Vorgänge einer richtigen Kenntnis der Wasserläufe bedarf, nimmt an ihr lebhaften Anteil. Überall aber findet man Strassen und Wege als Grenzen der Gerichte ${ }^{2}$ ). Sollte es unmöglich sein,

1) Vgl. darüber Richter, Über einen hist. Atlas (in der Festgabe für Krones) S. 6 f., Mitt. der geogr. Gesellsch. 1896, 532, Korrespondcnzbl. des Gesamtvereins 44, 74, Redlich in den Erläut. S. III, Sieger a. a. O. 262, Curschmann in der Hist. Vierteljahrschrift 11, $450 \mathrm{f}$. und 12, 14, $29 \mathrm{f}$., Karg.Bebenburg in den Forsch. zur Geschichte Bayerns 13, 253.

2) Zahlreiche Belege bieten die von Strnadt im Arch. 94 und 99 veröffentlichten Beschreibungen; vgl. auch den oben S. 596 erörterten Fall, wo der von mir vermutete Fehler auf der Karte kaum begangen worden wäre, wenn man die Strasse eingetragen hätte. 
Wenigstens bei den auf längere Strecken hinaus die Grenze begleitenden Strassen, sowie dort, wo die Überquerung der Strasse einen Punkt im Grenzzug ergibt, geeignete Signaturen in die Landgerichtsgrenze einzufügen? Das wird sich freilich ohne Begehung nicht durchführen lassen, aber gerade in der Nähe von Strassen ist ja ein solcher Lokalaugenschein verhältnismässig leicht zu erreichen. Selhr wünschenswert wäre, wenn auch die Grenzmarksteine, deren die Beschreibungen häufig gedenken, in der Natur aufgesucht und der Beuützer über ihr Vorhandensein unterrichtet würde. Das wird nicht überall durchführbar sein, vielleicht aber doch in der Nachbarschaft der Städte, deren Burgfried schon deshalb genauer und besser vermarkt zu sein pflegt, weil Material und Arbeitskräfte zur Herstellung deutlich gekennzeichneter Grenzsteine in der Stadt nicht fehlten. Linz besass allem Anschein nach in der Mitte des 17. Jahrhunderts durchlautend gezählte Grenzsteine im Umkreis seines Landgerichts, bei Freistadt ist dasselbe für 1743 bezeugt, die 1778 aufgenommene Beschreibung des Stadtgerichts Salzburg gibt von jedem Markstein an, welcherlei Zeichen er trug ${ }^{1}$ ). Unter solchen Umständen kaun es kaum grosse Schwierigkeiten machen, das etwaige Vorhandensein dieser alten Marken im Terrain festzustellen und die Grenzlinie darnach zu zeichnen oder zu kontrollieren²). Mir ist ein Fall bekannt, in welchem die ihm Jahr 1662 gesetzten salzburgisch-bairischen Marksteine noch genau an den Stelleu stehen, welche die damals aufgenommene Grenzbeschreibung angibt, und in welchem die Ausführung dieser Steine so einheitlich und vornehm gehalten ist, dass ihnen auch vom Standpunkt der Denkmälererhaltung sorgfältige Aufmerksamkeit geschenkt werden sollte ${ }^{3}$ ). Nachforschungen an Ort und Stelle verdient vielleicht auch der Landgraben oder Hörgraben, welcher an mehreren Stellen der einstigen bairisch-öster-

1) Für Linz s. Arch. 99, 291 f., für Freistadt Arch. 94, 289 Anm. 1, für Salzburg Mitt. der Gesellsch. f. Salzb. Landeskunde 28, $418 \mathrm{ff}$.

2) Strnadt erwähnt in den Erläut. S. 17 (vgl. Arch. 94, 587 Anm. 3), dass er am Teufelsbach bei Steyr noch einige Marksteine des Landgerichts der Stadt, »an welche in Steyr die Erinnerung völlig erloschen war ", ausfindig genacht habe.

3) Es sind das die ,Marksäulen" des Burgfrieds Mühldorf, die in dem salz. burgisch-bairischen Vertrag vom 13. Sept. 1662 (Zauner, Sammlung cler wichtigsten die Staatsverfassung Salzburgs betreffenden Urikunden S. 170 ff.) aufgezäblt sind, 27 am rechten, 23 am linken Ufer des Inn, beide Reihen mit besonderer Num. merierung. Ich sah und notierte mir im September 1907 einen grossen Teil dieser „Säulen`, welche in rotem Stein ausgeführt, den Boden um 1 m überragend, ganz gleichmässig mit dem salzburgischen und dem churbairischen Wappen geschmückt und mit denselben Nummern versehen sind, welche die Beschreibung an den betreffenden Stellen nennt. 
reichischen Grenze schon im 15. Jahrhundert nachweisbar'1) doch wohl auf eine alte Grenzbefestigung schliessen lassen dürfte.

So erweist sich die Eintrugung der durch Jahrhunderte sich forterbenden und fortentwickelnden Gerichtsbezirke als eine grosse und fruchtbare Arbeit. Richtig und gewissenhaft durchgeführt bietet sie dem Bearbeiter der Karte eine Fülle von geographischen Detailaufgaben, welche im einzelnen klein erscheinend durch die Beziehung zu bedeutenden geschichtlichen Fragen gehoben werden. Und bei weitem wicht alles, was der historische Geograph in diesem Zusammenhang erarbeitet, wird er auf der Karte niederlegen können, auch dann nicht, wenn ihm an wichtigen Punkten, durch Beigabe kleiner Nebenkarten ron grösseren Masstab die wünschenswerte Möglichkeit zur freieren Entfaltung gegeben wird. Eine grosse Menge der mit der Landgerichtskarte aufs eugste verbundeneu, dem Benützer ganz unentbehrlichen Ergebnisse, die er gewinnt, muss in Worte gekleidet, sie kann nicht blos im Kartenbild festgehalten werden. Diesem Mehrertrag. der sich kartographisch nicht buchen lässt, nü̈ssen die Abhandlungen dienen; möge es den Bearbeitern und der akademischen Leitung gelingen, den Raum, der diesen Abhandlungen gewidmet ist, wirklich im Sinne und im Dienste der Landgerichtskarte auszunützen; mögen sie nicht abschweifen auf Gebiete, die von ihr unabhängig und für sie entbehrlich sind, sondern im Rahmen der Abhandlungen oder im Auschluss an sie vor allem eine zuverlässige und praktisch eingerichtete Sammlung der Grenzbeschreibungen zustande bringen, welche für den Atlas eine Lebensbedingung ist. Neben diesen Abhandlungen und dieser Ausgabe werden die Erläuterungen ihren Platz als ein Behelf zu rascher Orientierung umso besser behaupten, je mehr sie sich strenger Kürze befleissen, je gewissenhafter sie, aller Übergriffe auf das Gebiet der Abhandlungen entkleidet und nur als Führer durch die Karte eingerichtet werden. So war es in der Hauptsache schon Richters Plan, als er die Grundzüge für die drei $Z$ weige des Unternehmens entwarf. Es wäre müssig darüber zu streiten, ob, wenn er selbst bis zum heutigen Tag über seinem Lieblingswerk hätte wachen dürfen, diese Grundzüge

1) Eingetragen sind der Landgraben und der Hörgraben auf $\mathrm{Bl} .9$ bei Powang und bei $O$. Aschau an der Grenze zwischen Ldg. Kogl und Wildeneck. Von den beiden in dem Urbar von Kammer 1550 (Erläut. S. 21, 2. Sp. u. Arch. 99 280). vorkommenden ,Landgraben " muss aber der eine nach Strnadt (Erl. a. a. 0.) in der nächsten Nähe von Unterach, der andere (Strnadt in den Mitt. des Instituts. 24, 435) nahe dem Rinderholz zwischen Frankenmarkt und Strasswalchen gesucht werden. 
etwa noch deutlicher in der Ausführung hervortreten würden, als es jetzt der Fall ist. Wenn aber seiner Künstlernatur die pedantische Strenge, die zur Leitung eines wissenschaftlichen Unternehmens gehört, von Anfang an fremd war, wenn es ihn von den Sorgen und Freuden eines den philologischen Mustern nacheifernden Editors immer wieder zur Fühlung mit Natur und Leben hinzog: die Erfahrung und die Liebe zu seinem Werk würde wohl auch ihn von der Notwendigkeit überzeugt haben, nicht nur selbst auf dem Wege zu bleiben, sondern auch seine Arbeitsgenossen strenge und genau auf dem engen Pfad festzuhalten. Sein ist das Verdienst, die Landgerichtskarte als das wichtigste und zunächst erreichbare Ziel historisch-geographischer Forschung in den österreichischen Alpenländern erkannt und ihre Durchführung gesichert zu haben, nun ist es an seinen Nachfolgern, das Werk rüstig zu fördern und glücklichem Abschluss zuzuführen. Möge ihnen das in solcher Art gelingen, dass ror allem die geschichtliche und geographische Erkenntnis unserer Heimat davon dauernden Nutzen ziehe; dann wird es auch an Früchten von allgemeinem wissenschaftlichen Wert gewiss nicht fehlen. 